\title{
Les paysages thérapeutiques de deux maternités d'Île-de-France
}

Clélia Gasquet-Blanchard, Alain Vaguet, Véronique Lucas-Gabrielli, Bruno Renevier et Elie Azria

\section{OpenEdition}

Journals

Édition électronique

URL : https://journals.openedition.org/rfst/522

DOI : $10.4000 /$ rfst.522

ISSN : 2492-3672

Traduction(s) :

The therapeutic landscapes of two maternity hospitals in lle-de-France - URL : https:// journals.openedition.org/rfst/752 [en]

Éditeur

Espaces et SOciétés (UMR 6590)

Référence électronique

Clélia Gasquet-Blanchard, Alain Vaguet, Véronique Lucas-Gabrielli, Bruno Renevier et Elie Azria, «Les paysages thérapeutiques de deux maternités d'Île-de-France », Revue francophone sur la santé et les territoires [En ligne], Miscellanées, mis en ligne le 13 novembre 2020, consulté le 04 mai 2021. URL : http://journals.openedition.org/rfst/522 ; DOl : https://doi.org/10.4000/rfst.522

Ce document a été généré automatiquement le 4 mai 2021.

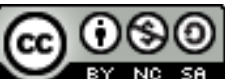

La Revue francophone sur la santé et les territoires est mise à disposition selon les termes de la Licence Creative Commons Attribution - Pas d'Utilisation Commerciale - Partage dans les Mêmes Conditions 4.0 International. 


\title{
Les paysages thérapeutiques de deux maternités d'île-de-France
}

\author{
Clélia Gasquet-Blanchard, Alain Vaguet, Véronique Lucas-Gabrielli, Bruno \\ Renevier et Elie Azria
}

Remerciements : Au CNFG, pour cette initiative, aux différentes personnes présentes lors des visites (Isabelle Siffert, Josianne Tanchou, Pétronille Reme-Harnay), aux chefs de services des deux maternités de nous avoir ouvert les portes de leurs services et aux professionnel.le.s des services visités.

A Jean-Benoît Bouron de Géoconfluences pour sa relecture de la première version du texte. Il a paru dans une forme courte à l'adresse suivante : http://geoconfluences.ens-lyon.fr

\section{Introduction}

\section{Positionnement théorique en géographie : appréhender les lieux comme espace de situation et d'interaction}

1 Beaucoup de disciplines de sciences humaines et sociales, comme la sociologie, l'anthropologie, l'histoire et la géographie, ont proposé des lectures des lieux des soins. L'histoire et la géographie des hôpitaux (Labasse, 19801) traitent de l'évolution de l'architecture hospitalière au fil du temps et des conséquences des diverses options choisies sur les usagers et les professionnels de santé. Si l'étude des lieux s'est longtemps focalisée sur la distribution spatiale de l'offre de soins, les perspectives de la géographie humaniste contemporaine s'intéressent au sens subjectif des lieux (Quel sens j'attribue à cet hôpital? Un sentiment de crainte, de confiance ?). On peut considérer dans cette perspective qu'il n'est de lieux que lorsqu'il se produit une relation sociale, une rencontre entre des personnes. C'est la raison pour laquelle certains chercheurs ont défini les non-lieux (Augé, 1992²) des lieux qui suscitent plus des croisements (comme les aéroports, les hypermarchés...) que des rencontres. Dans ce contexte l'hôpital est-il un non-lieu? 
2 La géographie a produit de nombreux travaux sur la santé en prenant comme entrée les lieux de santé (Gesler \& Kearns, 2002) et en leur donnant une portée générale qui interroge la place de l'identité, de l'expérience humaine, du corps, de l'environnement, de la culture. C'est ainsi que des lieux qui ont la réputation de soigner, d'agir sur la santé, de guérir (Lourdes, Bath, Epidaure...) ont été documentés par des chercheurs ${ }^{3}$ qui ont mis en avant le concept de " paysage thérapeutique "i, sur lequel nous revenons plus avant dans le texte (Hoyez, Gasquet-Blanchard, 2019). Au-delà de cette première étape, beaucoup de travaux interrogent désormais les lieux de soins, en se demandant s'ils sont tous également des lieux de santé. Les chercheurs anglophones ont beaucoup publié dans cette direction, en se demandant comment l'architecture d'un espace de soins pouvait prétendre avoir une dimension thérapeutique, voire améliorer la qualité des soins, la qualité de vie des personnes malades fréquentant ces lieux. Des éléments de paysages ont été pris en considération (la vue sur la nature, l'ouverture sur la ville, l'amélioration de l'éclairage, des couleurs,du repérage...) au fil des programmes de constructions/rénovations récents.

\section{Encadré 1 : Les hôpitaux en géographie}

Si les travaux des sciences sociales sur l'hôpital dans la ville sont nombreux, à l'échelle infra-hospitalière, les observations semblent plus rares (Granier, 2002, Vaguet et al, 2012). Pourtant, celles-ci permettent d'entrer rapidement dans les enjeux importants en termes de perception d'utilisation et de qualité des soins. Elle permet également de repérer la structuration du lieu et par là, de renseigner les espaces de pratiques des professionnels et des patients. Les recherches sur l'« humanisation » des soins à l'hôpital témoignent ainsi d'expériences d'amélioration, dont les plus répandues se traduisent souvent par une dimension matérielle du bâti (architecture, agencement, création d'espaces conviviaux) (Briand, Pary, 2011). Pourtant la dimension relationnelle avec les soignants, mais aussi avec l'usage des lieux est primordiale et c'est l'ensemble du paysage hospitalier qui fait partie du processus de guérison du patient. Ainsi, pour (Gesler et al, 2004) « la valeur thérapeutique d'un lieu de soins serait liée à la combinaison de ses dimensions physique, sociale et symbolique » (Gesler et al., 2004 ; Petit, 2010). C'est dans cette optique que l'on envisage de traiter la comparaison des deux maternités visitées. Pour ce faire, nous utilisons donc le concept de paysage thérapeutique (figure 1) en déclinant ses trois composantes. 
Figure 1 : L'environnement thérapeutique hospitalier

\section{Inpatient therapeutic environment}

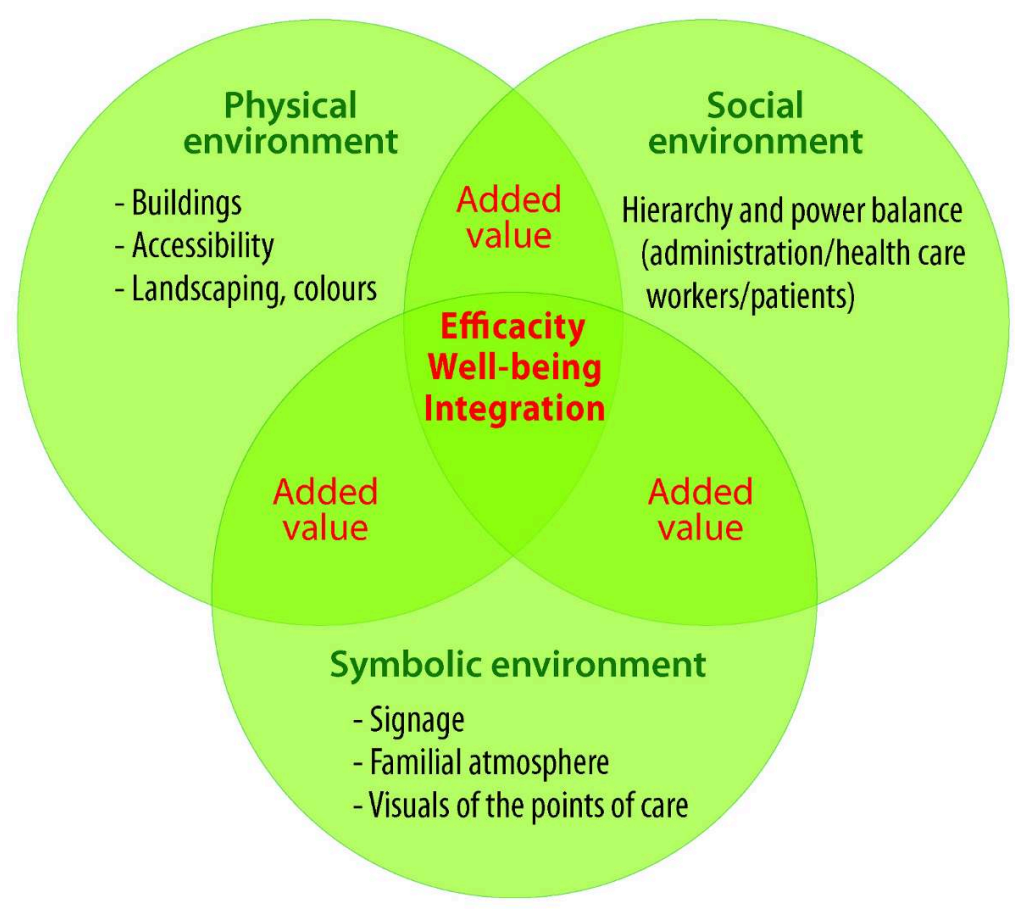

"Les trois composantes des paysages thérapeutiques s'ajoutent pour contribuer à caractériser ce qui constitue le bien-être dans un environnement thérapeutique : les éléments physiques, sociaux et symboliques. Ensemble qui participe à l'efficacité des soins, au bien-être du patient età son intégration » Source : Gesler (2002), Vaguet, Petit, Lefebvre (2012)

3 Malgré leur intérêt, ces améliorations se focalisent surtout sur les aspects physiques des bâtiments et ne règlent pas les difficultés rencontrées par les soignant.e.s dans leur exercice professionnel et les patient.e.s dans leur recours aux soins. Or, les lieux de soins fonctionnent mieux si une congruence harmonieuse s'établit entre le bâti et ses habitants et visiteurs (personnel lié aux soins, administratif, personnes malades, accompagnants). Un équilibre délicat entre le contrôle des coûts de fonctionnement et le bien-être des personnes utilisatrices des lieux de soin.

En replaçant dans leurs contextes sociohistoriques respectifs deux maternités visitées en 2018, la maternité du Groupe Hospitalier Saint-Joseph et celle du Centre hospitalier intercommunal de Montreuil, et en les mettant en regard, nous voulons contribuer à identifier certaines spécificités des interactions qui y existent, préciser sur quels symboles reposent les pratiques des usager.e.s et des professionnel.le.s et en quoi ces derniers participent à la différenciation sociale dans les rapports aux lieux (les pratiques spatiales des groupes).

\section{Encadré 2 : Méthodologie de l'article}

Cet article fait suite à deux visites commentées et collectives organisées, l'une fin 2018, l'autre début 2019, par une chercheuse sollicitée par la commission santé du Comité National Français de Géographie (CNFG), en vue d'ouvrir ses « terrains » de recherche parisiens à la réflexion collective. Ces ateliers ont été conduits essentiellement par un groupe de géographes travaillant sur les thématiques 
sanitaires. L'objectif de la commission santé du CNFG était de structurer une dynamique collective de réflexion scientifique articulant vécu collectif empirique et théorisation autour de notions usitées en géographie de la santé. Les visites se sont déroulées sur une demi-journée. Elles ont démarré avec l'accueil des chercheur.e.s par l'équipe de la maternité et la présentation de celles-ci, et se sont poursuivies par une visite des locaux commentée par un.e professionnel.le de santé ainsi que la rencontre avec les professionnel.le.s dans les différents services. En raison des temporalités de ce terrain particulier (collectif et très court), nous avons pris le parti de nous situer uniquement du point de vue des professionnel.le.s de santé et de ne pas axer notre propos sur les patientes et usagères de ces services. L'analyse et le propos se centrent volontairement et subjectivement sur le ressenti des chercheur.e.s durant la visite, les verbatims des visites commentées par les professionnel.le.s et leur analyse, puis des échanges entre chercheur.e.s et professionnel.le.s ayant eu lieu durant les discussions issues des restitutions. Cette méthodologie peu orthodoxe, se rapproche de « focus group in situ » et a nécessité des échanges de points de vue et ajustements nombreux pour l'écriture del'article.

Bien que l'objectif poursuivi n'ait pas été de lancer une recherche systématique, ces visites ont été enregistrées, retranscrites intégralement et analysées individuellement par les chercheur.e.s. puis ces éléments ont été croisés et discutés entre les chercheur.e.s pour produire une trame d'analyse. Dans un but de diffusion et échange des connaissances, les éléments d'observations ont été retravaillés et mis en lien avec la littérature scientifique sur le sujet et recontextualisées pour chacune de ces structures.

Ce travail a été délivré lors de deux réunions de restitutions de ces visites en avril et juin 2019 en vue de mettre en discussion les éléments présentés. Ces restitutions ont pris deux formes : celle d'un séminaire ouvert à l'ensemble du service dans la maternité du CHIM (Centre hospitalier intercommunal de Montreuil).Lors de ce séminaire, notre présentation n'était qu'une parmi d'autres durant une journée scientifique organisée par le chef de service et ouverte aux partenaires extérieurs (représentants médicaux, autres chercheurs, infirmières, aides-soignantes, sagesfemmes, médecins, etc.). Dans l'autre établissement (St Joseph), l'atmosphère était plus confidentielle, c'était celle d'un séminaire de service, sur la plage horaire du déjeuner. Il s'inscrivait dans l'organisation mensuelle du service qui prévoit un temps d'échange scientifique régulier au sein de l'équipe. L'assemblée était plus homogène - composée de médecins et sages-femmes du service - et plus restreint.

$5 \quad$ Nous avions la même présentation pour les deux services mais les modalités du cadre de présentation de la restitution ont été laissées à l'appréciation des chefs de services. Les débats issus de ces présentations ont participé à alimenter la réflexion développée dans cet article, ainsi que les allers-retours entre les auteur.e.s chercheur.e.s et professionnels de santé, durant l'écriture de cet article. De façon symptomatique, on a remarqué qu'ils ont assez systématiquement porté sur les paysages thérapeutiques différenciés entre structures du secteur public ou privé ${ }^{4}$, une des particularités du système français de soins, que nous discuterons au fil de l'article. Notre objectif consistait à nous interroger plus particulièrement sur l'agencement des lieux de soins 
et leur appropriation par les professionnels dans le contexte spécifique de la prise en charge de la grossesse en maternité. Mais ces maternités se situant et s'inscrivant dans des territoires, quelles relations à l'espace pouvait-on observer dans la pratique des lieux?

\section{Contextualisation des établissements visités}

\section{Situer les maternités dans leur contexte sociohistorique}

En France, le mémoire de Jacques Tenon, sur les hôpitaux de Paris (Tenon, 1788), pose les premières bases du modèle de l'hôpital pavillonnaire (comme l'Hôtel Dieu, illustré photographie 1). L'arrivée de l'hygiénisme et l'inspiration des hôpitaux américains participe à l'avènement des cités jardins hospitalières (comme l'hôpital EdouardHerriot)(photographie 2) à la place des anciens hôpitaux pavillonnaires, réaménagés à cet effet. L'hôpital Saint-Joseph témoigne de cette période (encadré 3).

Photographie 1 : L'Hôtel Dieu, hôpital historique et pavillonaire

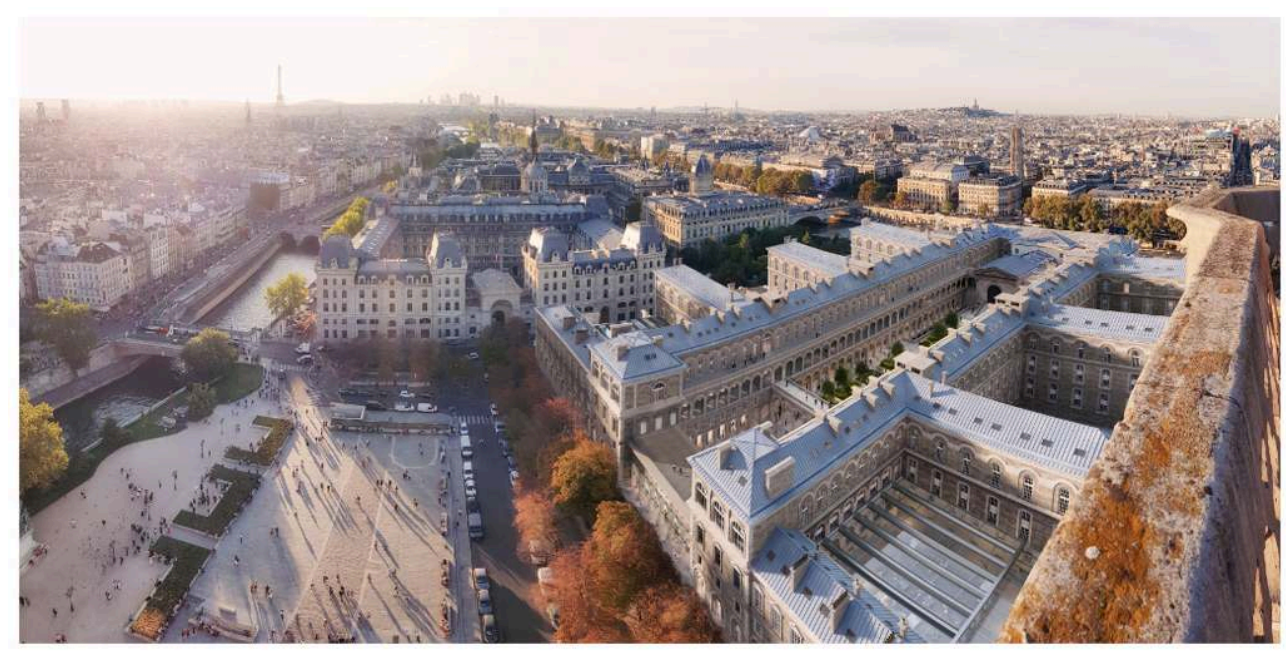

Source : hôtel dieu (C) AAD. Site consulté le 29 juin 2020 : https://chroniques-architecture.com/hoteldieu-anne-demians/ 
Photographie 2 : L'Hôpital Edouard Herriot, l'avènement des cités jardins

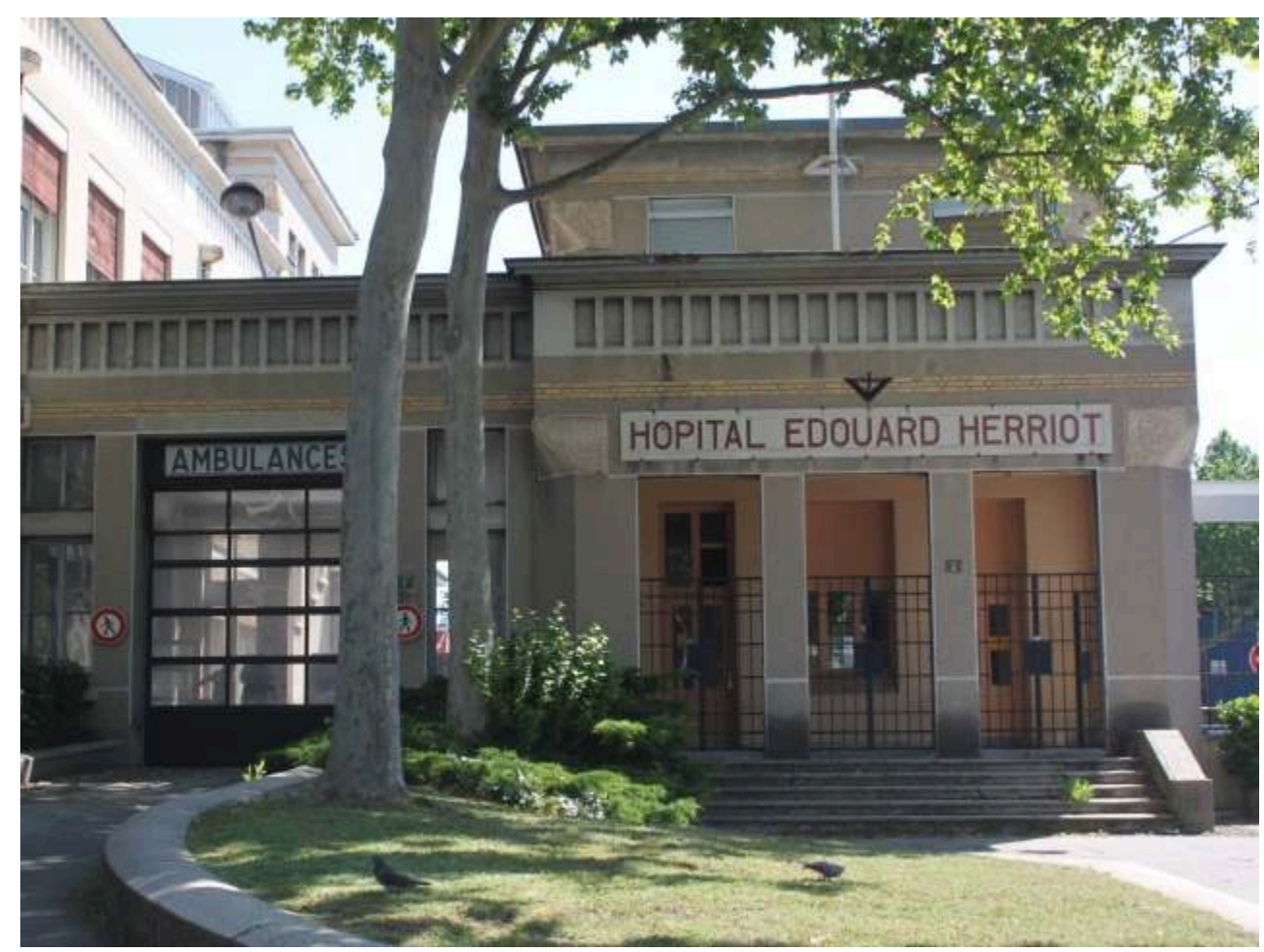

Source : Site consulté le 7 juillet 2020 https://www.lyonmag.com/article/106557/coronavirus-uneintersyndicale-de-l-hopital-edouard-herriot-reclame-un-depistage-systematique

7 Avec les progrès de l'antisepsie, le regroupement des bâtiments et des services devient possible et se développent les premiers hôpitaux-blocs, dans les années 1930 aux ÉtatsUnis puis en Europe, comme l'Hôpital Beaujon à Clichy (1932) représenté sur la photographie 3. Cette évolution technique participe à l'emploi de nouveaux matériaux : béton, ascenseurs, systèmes artificiels d'éclairage et de ventilation... Ces nouveaux bâtiments se développent dans une verticalité qui «invite à une réflexion sur la forme architecturale et sur la disposition des services et des circulations des patients, des visiteurs, de la logistique et du personnel» [Fermand 2000]. Dès lors le modèle de l'hôpital-bloc va poser clairement la question de l'efficacité et du confort pour les patients et les professionnels. 
Photographie 3 : L'Hôpital Beaujon, symbole des premiers hôpitaux blocs

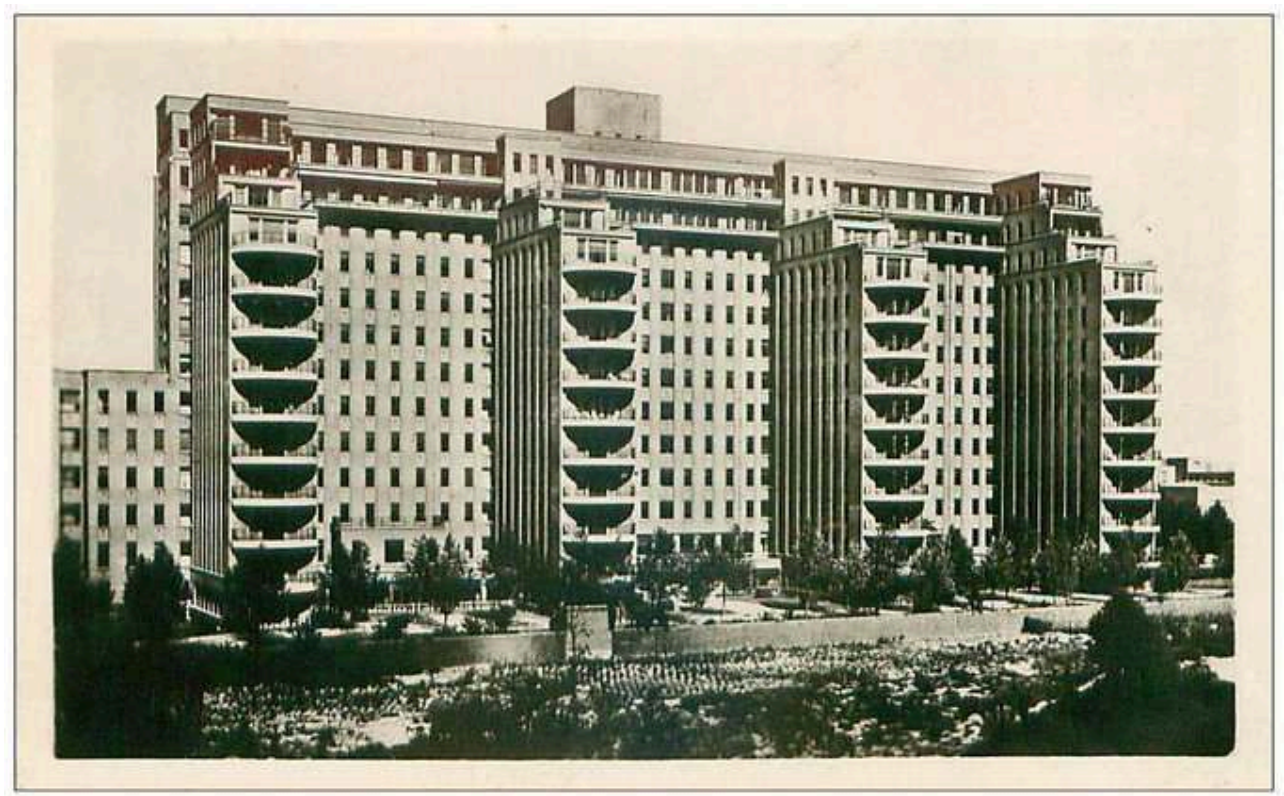

Source : https://twitter.com/Memoire2cite/status/1145658231219527680/photo/1 Extrait de l'Illustration 1935. Site consulté le 29 juin 2020

Photographie 4 : L'hôpital intercommunal de Montreuil

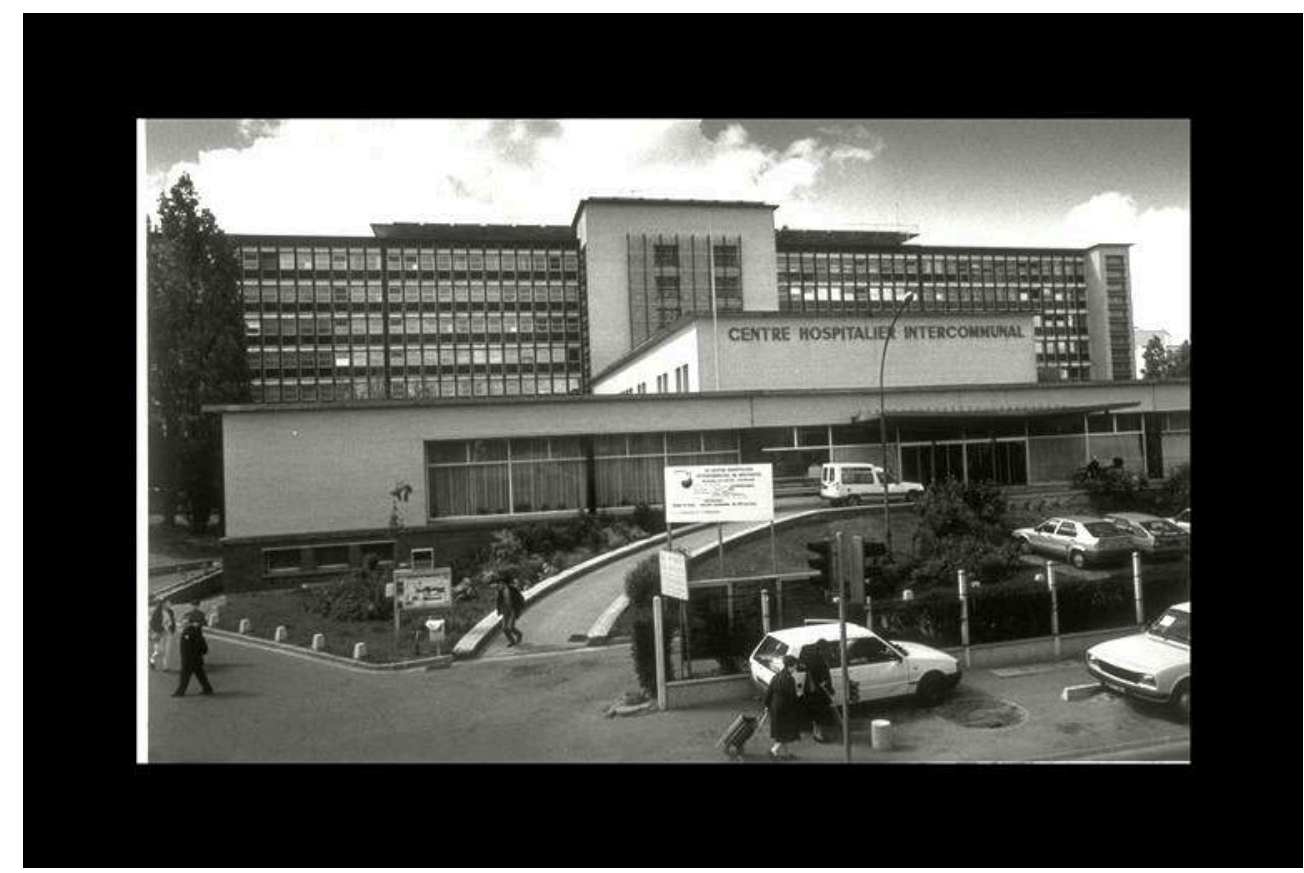

Photographie mise en ligne pour les 50 ans de l'hôpital en 2015. @ Site de l'hôpital de Montreuil

8 Dans le même temps, « la planification et la création des cartes sanitaires vont permettre de mieux contrôler le développement de l'infrastructure hospitalière sur le territoire national et de rationaliser l'organisation des services. Cette politique aboutit vers la fin des années 1960 à l'industrialisation des constructions publiques, notamment dans leur conception : objectif étant de rationaliser les circulations et les liaisons fonctionnelles tout en maitrisant les coûts» [Fermand 2000]. C'est le cas de la maternité du Centre hospitalier 
intercommunal de Montreuil (encadré 2), intégrée au sein d'un centre hospitalier intercommunal (1965). Il propose des prestations médico-chirurgicales de proximité pour les disciplines dites "courantes" : médecine, chirurgie générale et obstétrique.

9 Dans les faits le bilan (d'une rationalisation via une industrialisation des services de santé et de leur fonctionnement) de la période des trente glorieuses,reste mitigé, car si ces évolutions améliorent l'accès aux soins, la réflexion sur les logiques de fonctionnement hospitalier restent peu développées : « les besoins de l'hôpital priment, au stade conceptuel, sur ceux des futurs malades. L'étude démographique, les attitudes, les modes de vie et de consommation sont à peine évoqués, voire éludés, en faveur d'une efficacité d'abord budgétaire » [Labasse 1980, p. 102]. Dès lors, "l'aspect standard a semblé inhumain aux usagers et les professionnels de santé ont critiqué l'absence de lumière de certains locaux » [Fermand 2000], montrant les limites du modèle de «l'hôpital puzzle $»^{5}$ et un manque de vision stratégique à long terme en France.

10 Dans les années 1980, l'ouverture des commandes publiques et la politique de décentralisation ainsi que le développement du lien ville-hôpital impliquent la construction de bâtiments plus ouverts sur l'extérieur, comme c'est le cas des deux maternités visitées. En parallèle, depuis les années 1970, une politique de sécurisation des naissances suit son cours autour de trois plans de périnatalité successifs qui induisent une rationalisation des choix budgétaires.L'organisation des soins périnataux prévue par les différents décrets ministériels a participé à une réduction importante du nombre de maternités, bien qu'en moyenne le temps parcouru par les femmes pour se rendre à la maternité pour accoucher n'ait pas changé depuis 2003 (Source https:// drees.solidarites-sante.gouv.fr/IMG/pdf/er1031.pdf) .

Encadré 3 : Des maternités situées dans des contextes socio-historiques différenciés :

Le Groupe hospitalier Paris Saint-Joseph est une fondation privée issue de la fusion de différents hôpitaux

L'hôpital Saint-Joseph résulte de la construction de différents pavillons entre 1878 et 1910 dans Paris, suite à l'initiative de Monseigneur d'Hulst. Grâce à la générosité d'un groupe de catholiques laïcs, un terrain est acquis pour y construire l'hôpital Saint-Joseph. A cette date l'établissement est dirigé et géré par la congrégation des Sœurs de Saint Vincent de Paul, ce jusqu'en 1958. En 1976, l'hôpital est admis à participer au service public hospitalier, en lien avec l'hôpital Broussais. Il devient une fondation en 1977. Une politique de modernisation des bâtiments débute en 1995 et se poursuit de nos jours. Celle-ci va de pair avec la fusion avec les hôpitaux Saint-Michel et Notre Dame de bon secours.

Source : https://www.hpsj.fr/qui-sommes-nous/historique/

Le Centre hospitalier intercommunal André Grégoire de Montreuil voit le jour grâce à une politique d'aménagement d'aménité pour un bassin de population d'une banlieue populaire.

Cet hôpital public est un projet porté par la volonté de 9 communes de Seine-SaintDenis et limitrophes (Bagnolet, Fontenay-sous-Bois, Les Lilas, Montreuil-sous-Bois, Noisy-le-Sec, Romainville, Rosny-sous-Bois, Villemomble, Vincennes). Proposant 
un établissement de santé à leur population (400.000 habitants) le Centre Hospitalier de Montreuil ouvre le 5 juillet 1965 son premier service : la maternité. Source : http://www.chi-andre-gregoire.fr/lhopital-fete-ses-50-ans/

Photographie 5 : Maternité Saint-Joseph

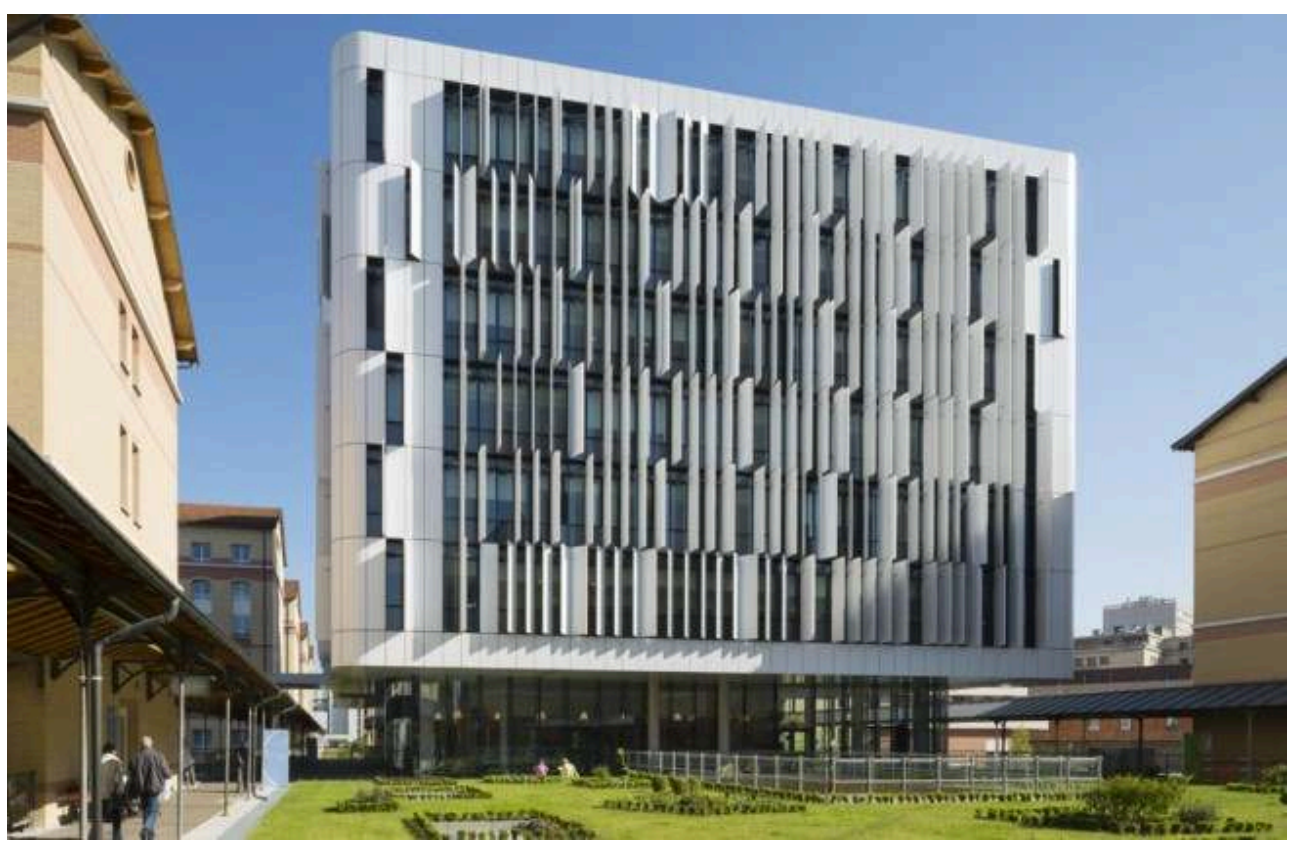

Source : Site internet consulté le 7 juillet 2020: https://www.kopines.com/etablissements/maternitenotre-dame-de-bon-secours-paris-saint-joseph/ 


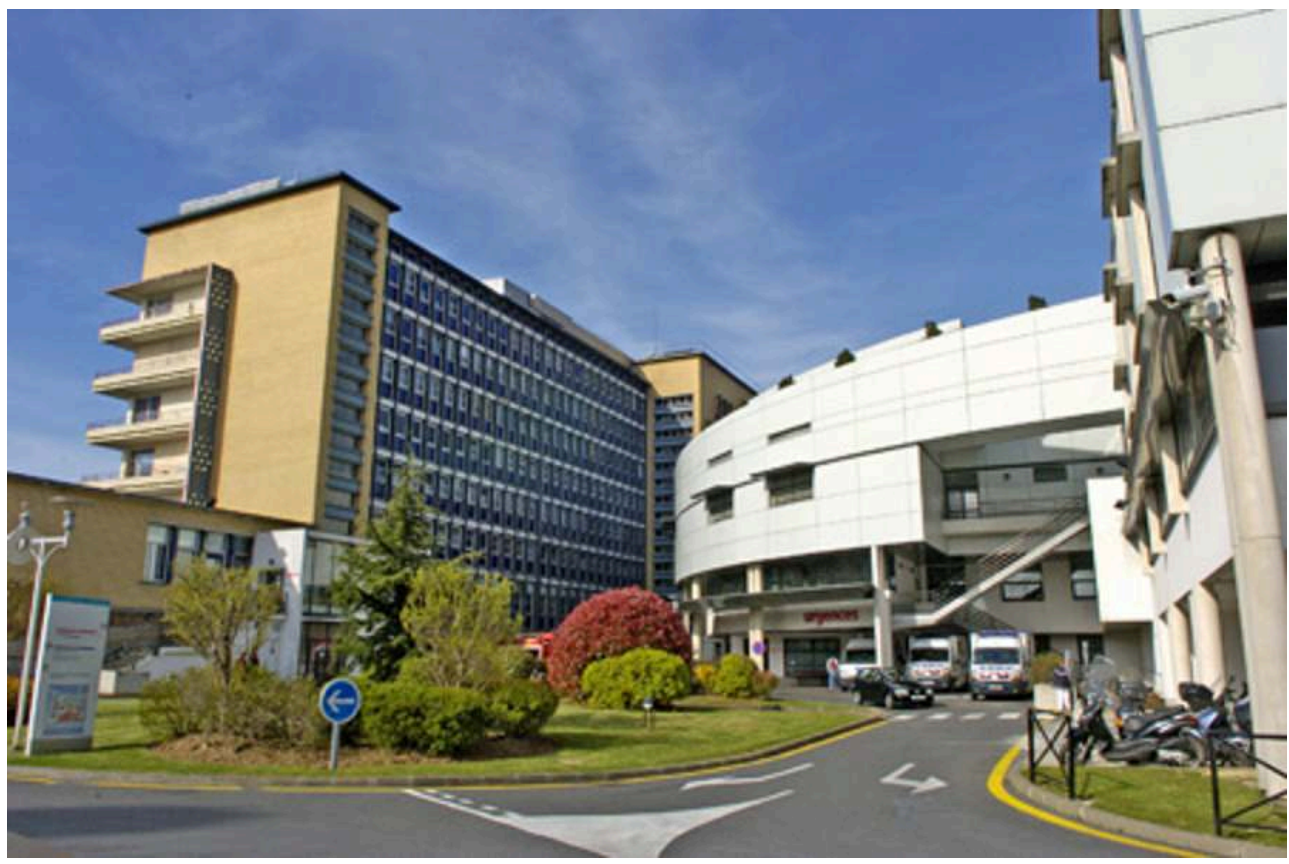

Source : Site internet de l'hôpital, consulté le 7 juillet 2020 @

Deux prises de vues qui soulignent en toile de fond une appropriation différenciée des espaces, plutôt dédiés à la promenade à Saint-Joseph et à la circulation des véhicules de service à Montreuil.

Inscrites toutes les deux dans des contextes historiques et architecturaux très différents les deux bâtiments de ces maternités sont néanmoins pensés dans une optique d'ouverture sur l'extérieur, au cœur de leurs complexes hospitaliers respectifs (articulation du moderne et de l'ancien pour SaintJoseph dont le passé d'hôpital pavillonnaire est utilisé pour un aménagement piéton des déplacements entre bâtiments à l'intérieur de l'enceinte de l'hôpital, les véhicules circulant autour. A Montreuil le nouveau bâtiment de la maternité s'encastre dans des constructions s'échelonnant des années 70 à 2000 privilégiant des accès aux véhicules de services aux différents bâtiments. Les usages piétons, se font par l'extérieur de l'enceinte de l'hôpital ou par des galeries reliant les bâtiments entre eux. En comparant ces deux prises de vues des maternités visitées, on constate au premier regard une unicité de forme, qui s'estompe toutefois lors de l'analyse des deux photographies.

S'opposent en réalité, sur la gauche des clichés, une barre d'immeubles à des pavillons réaménagés mais dont le style a été préservé ; de la même manière, les bâtiments modernes blancs s'opposent par leur ouverture : I'un en arrondi, sans beaucoup d'ouverture, l'autre est très ouvert. De même, l'espace vert au premier plan sur les deux clichés est dans les deux cas visuellement appropriables, mais plus ou moins bien mis en valeur : l'un est traversé d'une allée piétonne (espace vert de promenade et d'ornement) et l'autre d'un accès véhicule sans possibilité pour les piétons d'accéder à la pelouse (espace vert non appropriable et mis à distance par l'accès véhicules hospitaliers).

\section{Mise en regard des contextes sociaux des deux maternités}

11 Les maternités visitées sont situées en Île-de-France. L'une est située dans la commune de Montreuil, une ville populaire ${ }^{6}$ du département de la Seine-Saint-Denis (département le plus pauvre d'île-de-France et de métropole), comme l'illustre la carte 1. C'est une maternité publique de type 3 (le niveau de technicité le plus important, notamment en termes de prise en charge néonatale). L'autre, Saint-Joseph est de type 2b. Elle est située à Paris dans le $14^{\mathrm{ème}}$, un arrondissement favorisé, classé dans la typologie de l'IAU illustrée par la carte 1 comme un « espace aisé de l'Ouest ». C'est une maternité privée ${ }^{7}$ à but non lucratif qui participe à la mission de service public en proposant des consultations sans dépassement d'honoraires, pouvant néanmoins proposer des formules d'hôtellerie premium occasionnant un surcoût à destination de patients internationaux ${ }^{8}$. 


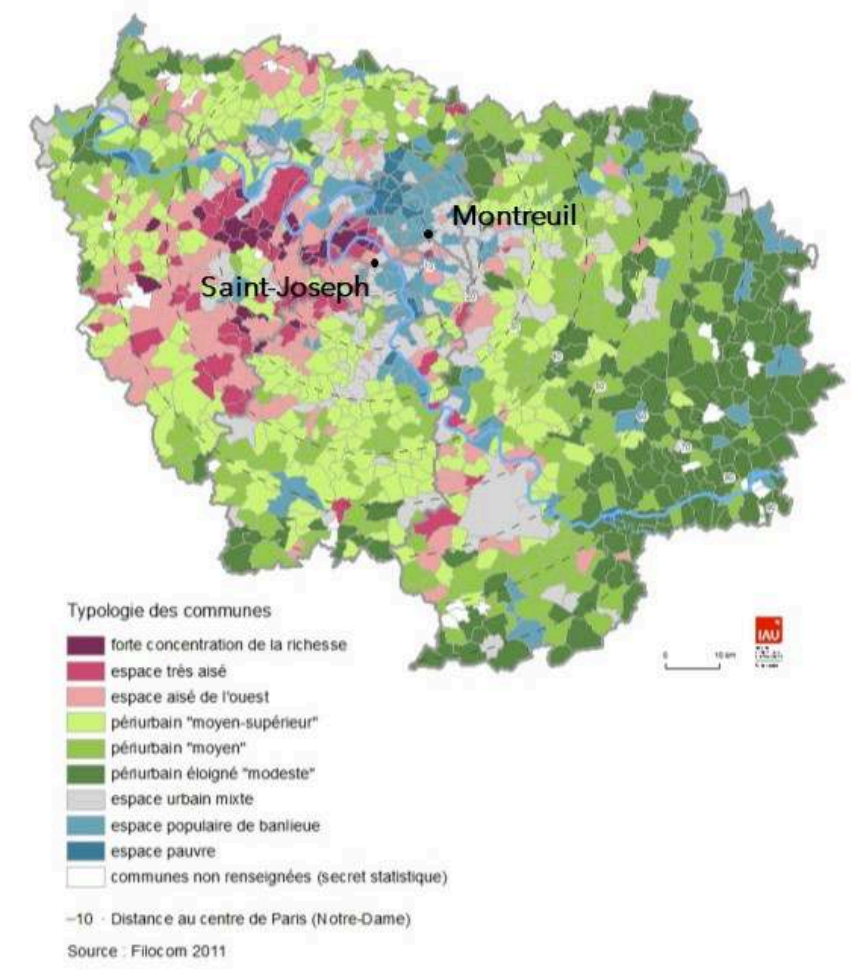

Source : IAU Île-de-France, 2013, Géographie sociale et habitat en Ile-de-France, Evolutions 2001-2011 ; Septembre 2013, 93p.

Située dans le Sud de Paris, la maternité Saint-Joseph est facilement accessible en transport en communs (tram, métro, bus). Celle de Montreuil est accessible en bus, mais surtout en voiture, comme l'illustre le croquis 1 réalisé par Île-de-France Mobilités / Latitude-Cartagène en juillet 2017. Il faut dire que l'hôpital Saint Joseph est situé dans la première zone de densité et d'accessibilité car il est situé dans Paris intramuros alors que la maternité de Montreuil se situe au milieu d'une enclave du Réseau Express Régional (RER). Au moment de la visite, l'accès de cette dernière est difficile car son environnement immédiat est en travaux. En effet, l'hôpital se trouve au cœur de la dynamique d'aménagement du Grand Paris, avec l'arrivée prochaine du métro via le prolongement de la ligne 11 qui situera, dans une à deux décennies, l'hôpital à 10-12 minutes de Paris9. À l'échelle des villes, de nombreux travaux montrent que l'hôpital est un pôle de référence, de formation universitaire, important pourvoyeur d'emplois (Fleuret, 2012). Mais la communication sur cet aspect positif n'est que peu développé. Dès lors, pour vaincre cette stigmatisation, les établissements comme l'hôpital de Montreuil, cherchent à rétablir une vision plus globale de la situation, celle d'un établissement qui offre des services pointus et qui s'adapte aussi à la population de l'Est parisien. Un établissement attentif à accompagner les mutations prévisibles de son bassin de population, montrant comment « les autorités administratives et sanitaires ont pour volonté de faire de l'hôpital un objet urbain» (Labasse, 1980) et situant l'hôpital dans une politique urbaine au cœur des grandes options d'urbanisme (Clémençon, 2002). 


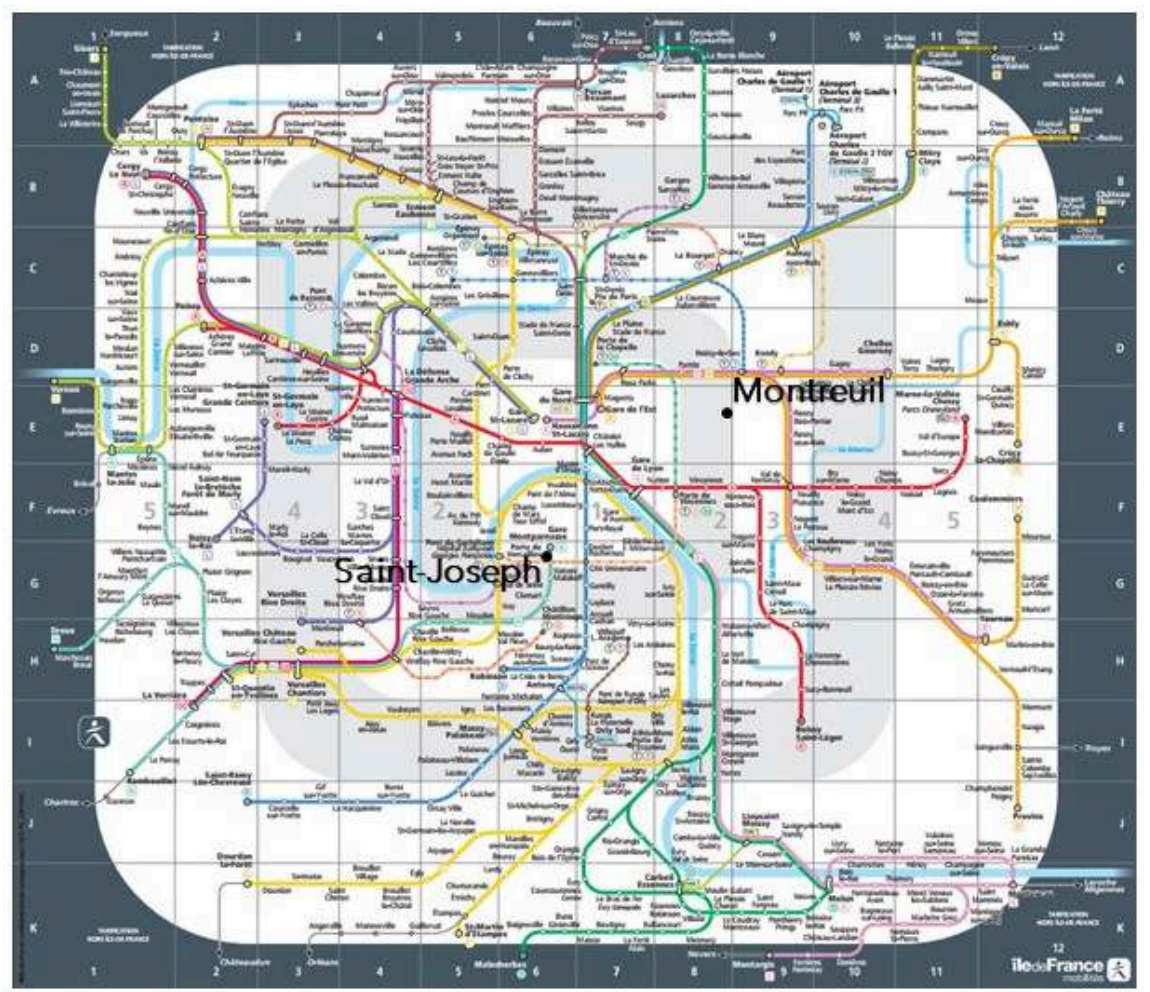

Source : Plan régional des transports d'île-de-France (île-de-France Mobilités / Latitude-Cartagène juillet 2017)

13 Pour l'hôpital de Montreuil, au cœur d'enjeux liés à l'aménagement du grand Paris, l'échelle urbaine est cruciale : La maternité se retrouvera bientôt, en raison de temps d'accès plus courts, en concurrence directe avec Trousseau, Necker, Port Royal et d'autres prestigieux hôpitaux parisiens. Beaucoup d'éléments non médicaux semblent interférer sur l'évolution et le type d'offre de soins. Ainsi, pour la maternité intercommunale de Montreuil, au cœur d'un bassin de population en gentrification, se pose par exemple la question de l'évitement du CHIM par certain.e.s patient.e.s, notamment les plus aisées, au profit des maternités prisées de l'Est parisien ou de proche banlieue comme la Maternité des Lilas, la maternité des Bleuets ou encore les Diaconnesses (pour leur approches physiologiques de la grossesse) ou inversement les grandes maternités de haute technicité de l'AP-HP (comme Trousseau ou Robert Debré). C'est actuellement le cas d'une grande partie des femmes résidant dans le Bas Montreuil ${ }^{10}$. Ce phénomène est lié notamment à des représentations négatives que peuvent avoir certaines populations d'un hôpital de service public non universitaire, particulièrement celles de classe moyenne habitant le centre-ville et souhaitant notamment des accouchements moins médicalisés. Ce phénomène se couple aux représentations stigmatisantes liées à l'importante fréquentation des populations paupérisées, vulnérables, et migrantes.

14 Bien que le quartier de Saint-Joseph, aux portes de Paris, connaisse à l'inverse une dynamique de paupérisation de certains îlots, la localisation de la maternité la situe dans un bassin de recrutement d'une population plus aisée que la maternité de Montreuil (les patientes de Saint-Joseph proviennent notamment des 14 et $15^{\text {ème }}$ arrondissements, et des communes alentours) et n'implique pas du tout les mêmes 
enjeux face aux politiques d'urbanismes actuelles, puisque par sa localisation intramuros Saint-Joseph deviendra à terme une maternité centrale du Grand Paris, dans l'espace favorisé de la capitale comme l'illustre la carte $n^{\circ} 1$.

15 Relativement à leur activité, ces deux maternités sont comparables en termes d'activités et de pratiques: environ 4000 accouchements à Montreuil dont 19,6\% de césariennes, et environ 3500 à Saint-Joseph, dont $17 \%$ de césariennes. Elles ont toutes deux emménagé dans un bâtiment neuf ouvert en 2011 à Saint-Joseph et en 2017 à Montreuil.

16 Les localisations différentielles influencent, de fait, la prise en charge d'une catégorie sociale de femmes propre à chacune des deux maternités. La maternité de Montreuil, bâtiment inspiré par les constructions standardisées des années 1960, se présente comme recevant de longue date des femmes en situation de précarité voire de grande précarité : sur 17000 consultations annuelles d'obstétrique, 700 femmes suivies sont bénéficiaires de l'AME - Aide Médicale d'Etat ${ }^{11}$. En raison d'un nombre conséquent de patientes victimes de violences, une consultation sage-femme est dédiée à cette question, et une gynécologue spécialisée dans la reconstruction vulvaire des femmes excisées a été recrutée. Plus récemment une unité d'accompagnement personnalisée à destination des femmes en situation de vulnérabilité a été mise en place également. La maternité est adossée au Centre d'IVG (9 places de bloc par semaine) en lien avec le planning familial, permettant la réalisation de 1100 IVG par an. Cet acte n'est pas réalisé à la maternité de Saint-Joseph, qui renvoie, vers l'Institut Mutualiste Montsouris, lorsque l'on pose question à l'accueil des consultations. En raison d'un nombre important de femmes allophones, les professionnels de santé font également régulièrement appel à une entreprise d'interprétariat. Enfin, une unité kangourou dispose dans le service de néonatologie de lits d'hospitalisation mère/enfant.

17 De son côté, la maternité de Saint-Joseph, s'inscrit dans le Groupe hospitalier Paris Saint-Joseph, relevant d'une fondation. Parmi ses 3500 accouchements annuels, 609 séjours de grossesses à hauts risques (831 à Montreuil) et d'hospitalisation de médecine obstétricale sont pris en charge à Saint-Joseph. En unité de soins intensifs, 264 nouveau-nés sont pris en charge (377 à Montreuil) et 486 en médecine néonatale (210 à Montreuil).

18 Après avoir rappelé rapidement l'arrière-plan problématique et théorique, remis en contexte des maternités visitées, nous nous appuyons maintenant sur le concept de paysage thérapeutique pour présenter les principales conclusions résultant de ces visites.

\section{La maternité comme paysage thérapeutique}

\section{Environnement physique entre sobriété et fonctionnalité à Montreuil et chaleur d'un accueil architecturalement travaillé à Saint-Joseph où transparait la symbolique des lieux}

19 La première composante du paysage thérapeutique qui peut être évoqué est l'environnement physique. "Il concerne ce qui semble le plus simple à mesurer et à caractériser par les cinq sens du chercheur, particulièrement son sens visuel: surfaces, luminosité, propreté, vue sur la nature... mais aussi de tous les autres éléments permettant la 
caractérisation du site ou du service (accessibilité, relégation, centralité...) " (Vaguet, Petit, Lefebvre 2012). De ce point de vue, si les deux bâtiments visités sont récents, clairs, la signalétique plus classique et sobre à Montreuil soulève un point de vigilance. Dans l'ensemble la dimension physique, sur le versant hôtelier, donne un avantage certain à des établissements comme Saint-Joseph, comparativement à la plus stricte frugalité du site de Montreuil, orienté vers la seule fonction socio-médicale. Ceci est bien illustré dès l'entrée à Montreuil, par le versant administratif (prise de ticket immédiat photographie 7) quand on entre par l'entrée principale de l'hôpital, le positionnement face à l'entrée du secrétariat (photographie 9), grande largeur des couloirs (photographie 10), participent à une entrée fonctionnelle dans les lieux. L'entrée propre de la maternité (photographie 11) à l'arrière de l'hôpital, s'orne de sculptures attractives, souhaitant refléter la diversité des femmes y accouchant.

Photographies 7 et 8 : Fonctionnalités des lieux de soins de l'hôpital public de Montreuil ${ }^{12}$

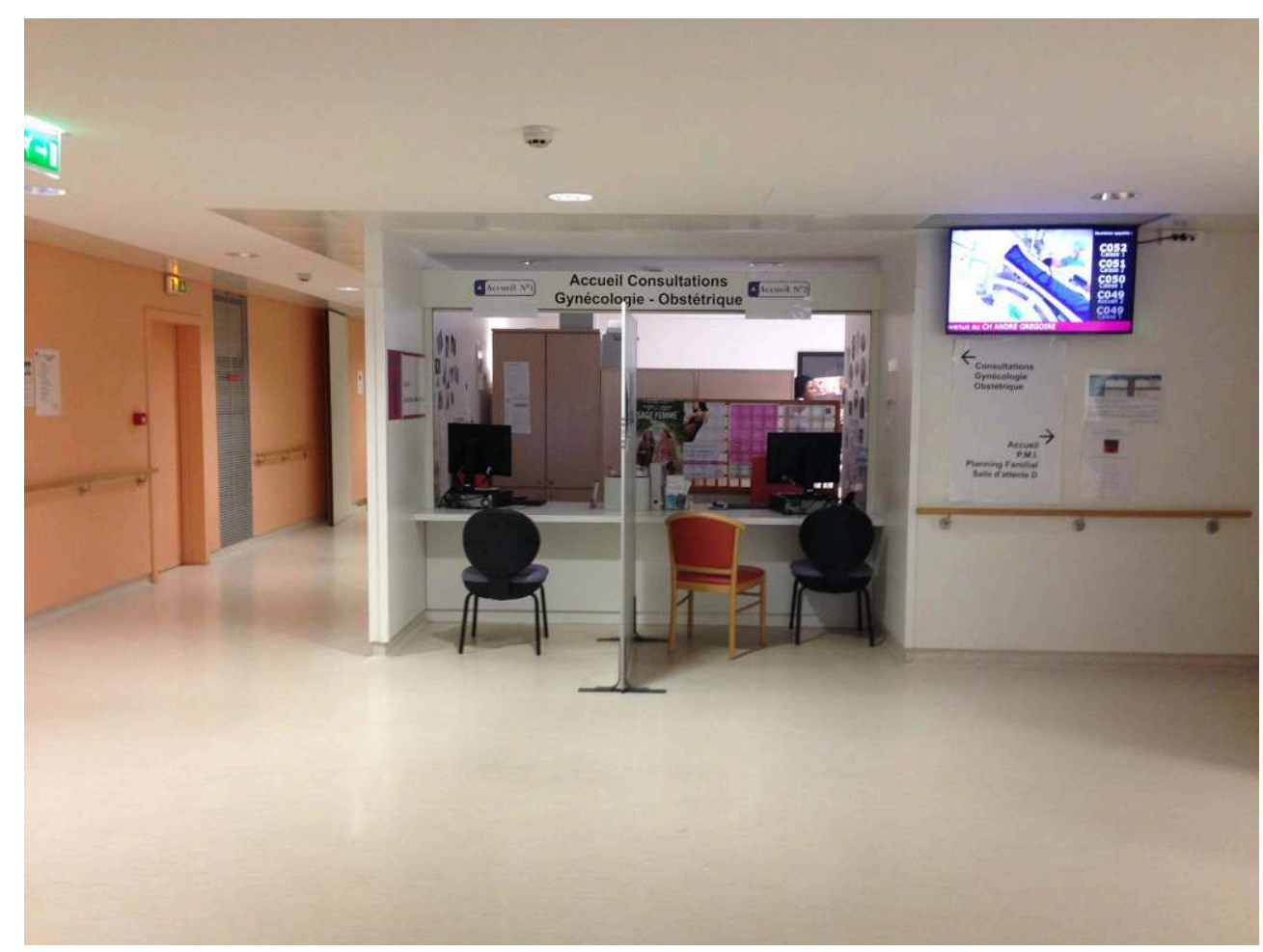




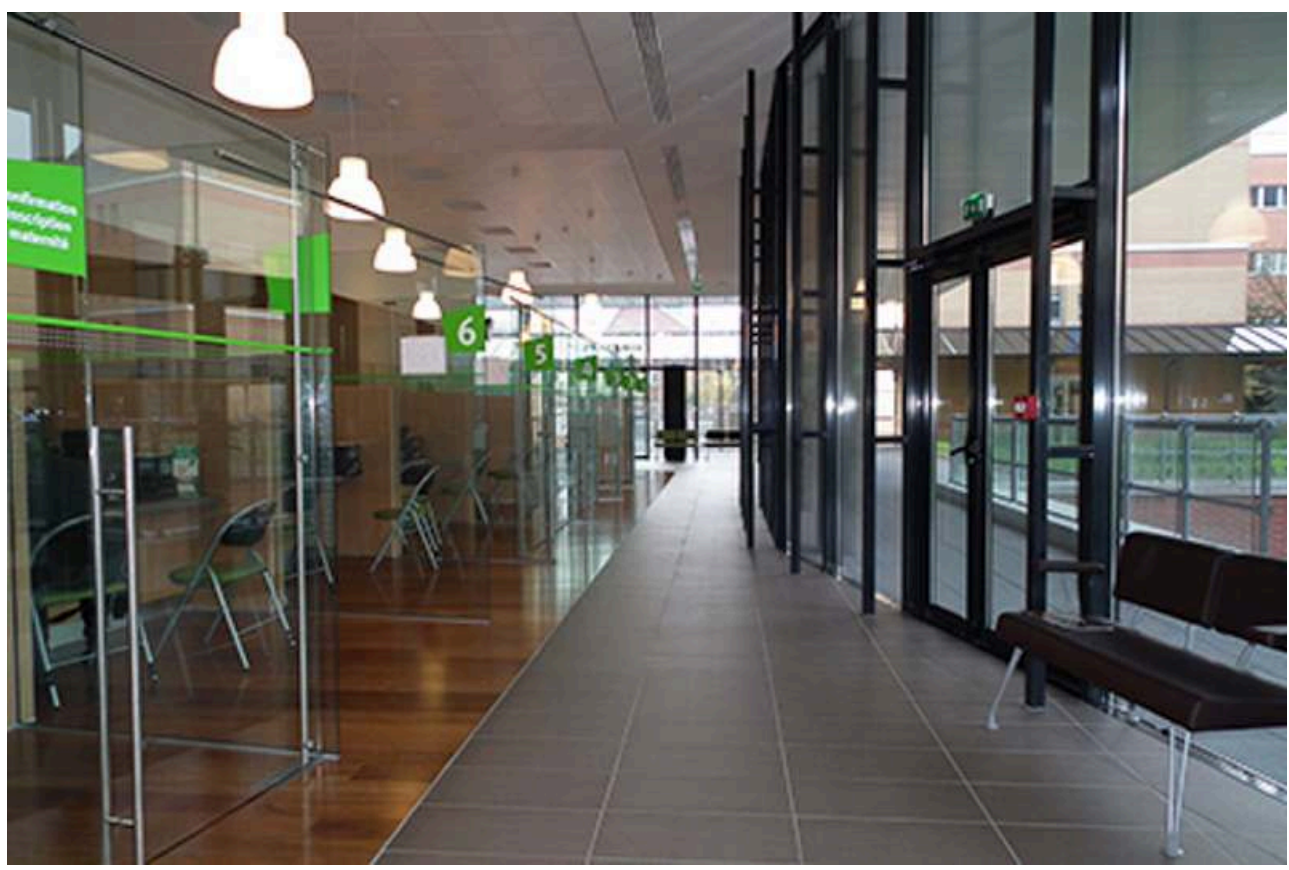

Un espace d'accueil et d'inscription vaste, ouvert et aéré, grâce à une organisation avec des boxes d'inscription en verre à Saint-Joseph (photo 8) et plus cloisonné à Montreuil mais moins confidentiels, puisqu'ouverts, et séparé par une simple cloison

Photographies 9 et 10 : Fonctionnalités des lieux de soins de l'hôpital public de Montreuil.

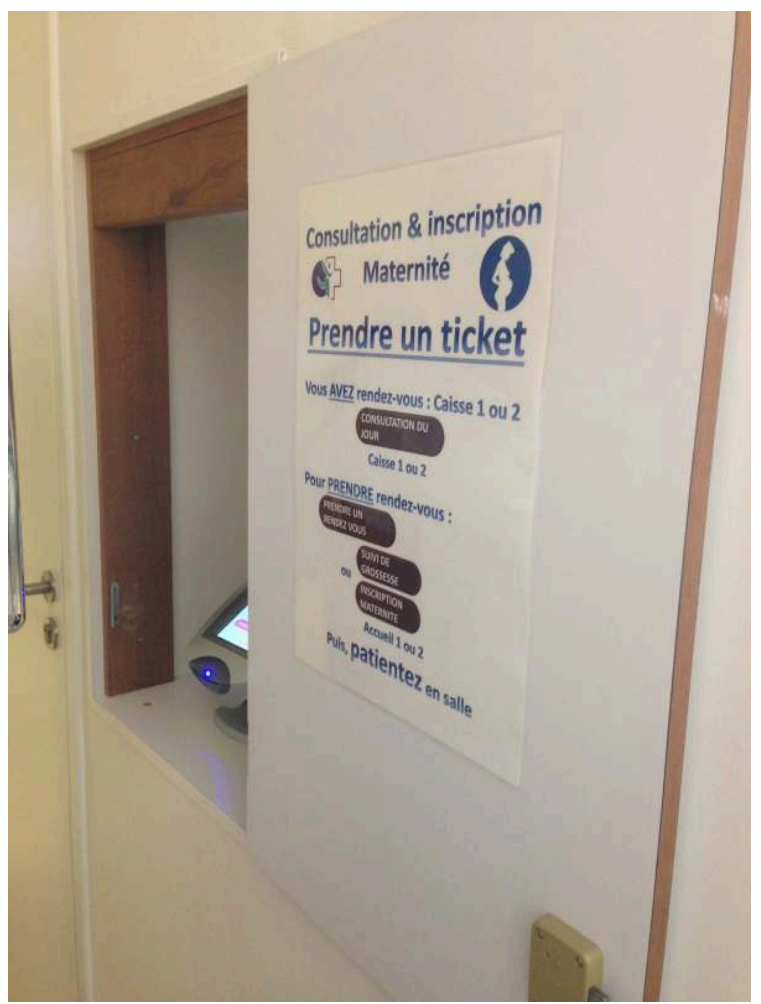




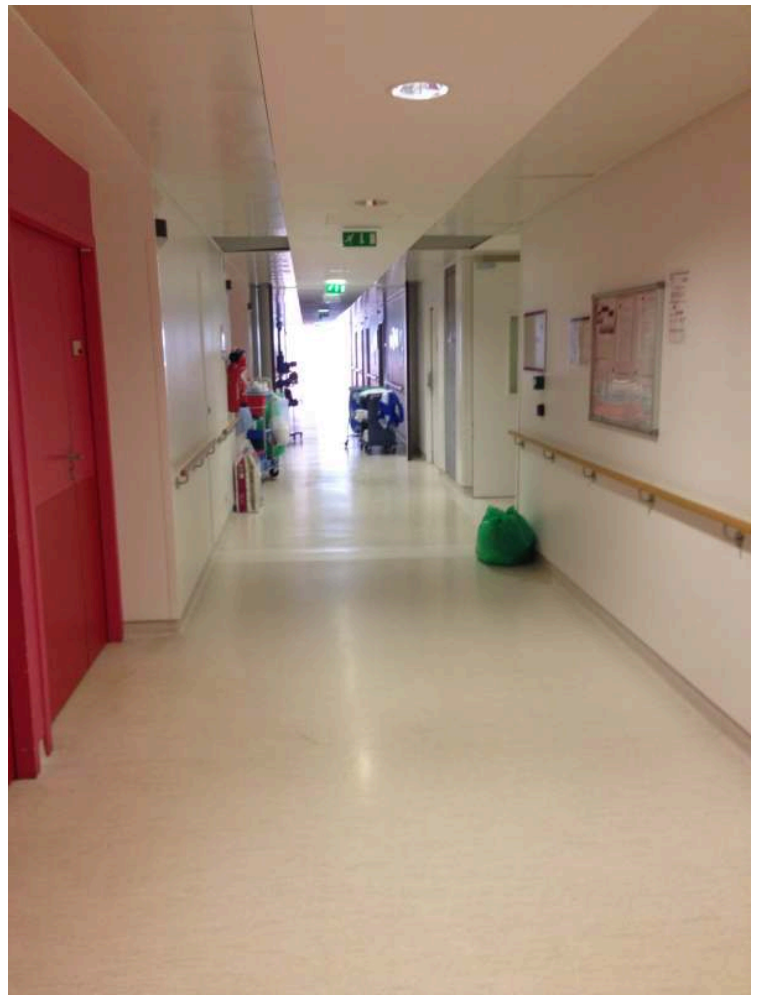

Photographie 11 : L'entrée de la maternité de Montreuil : symbole de diversité

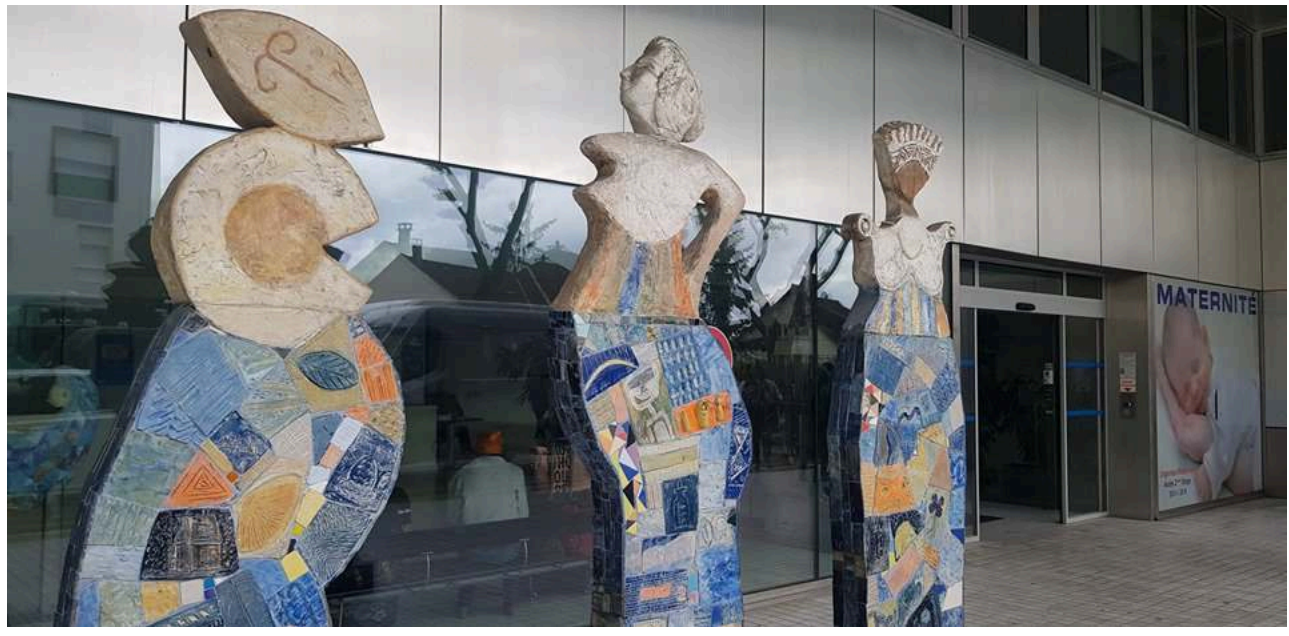

Source : Site internet consulté le 7 juillet 2020: https://twitter.com/EDH_LouisLareng/status/ 1143052180427030528 
Photographies 12 et 13 : Sobriété de l'affichage à l'hôpital public
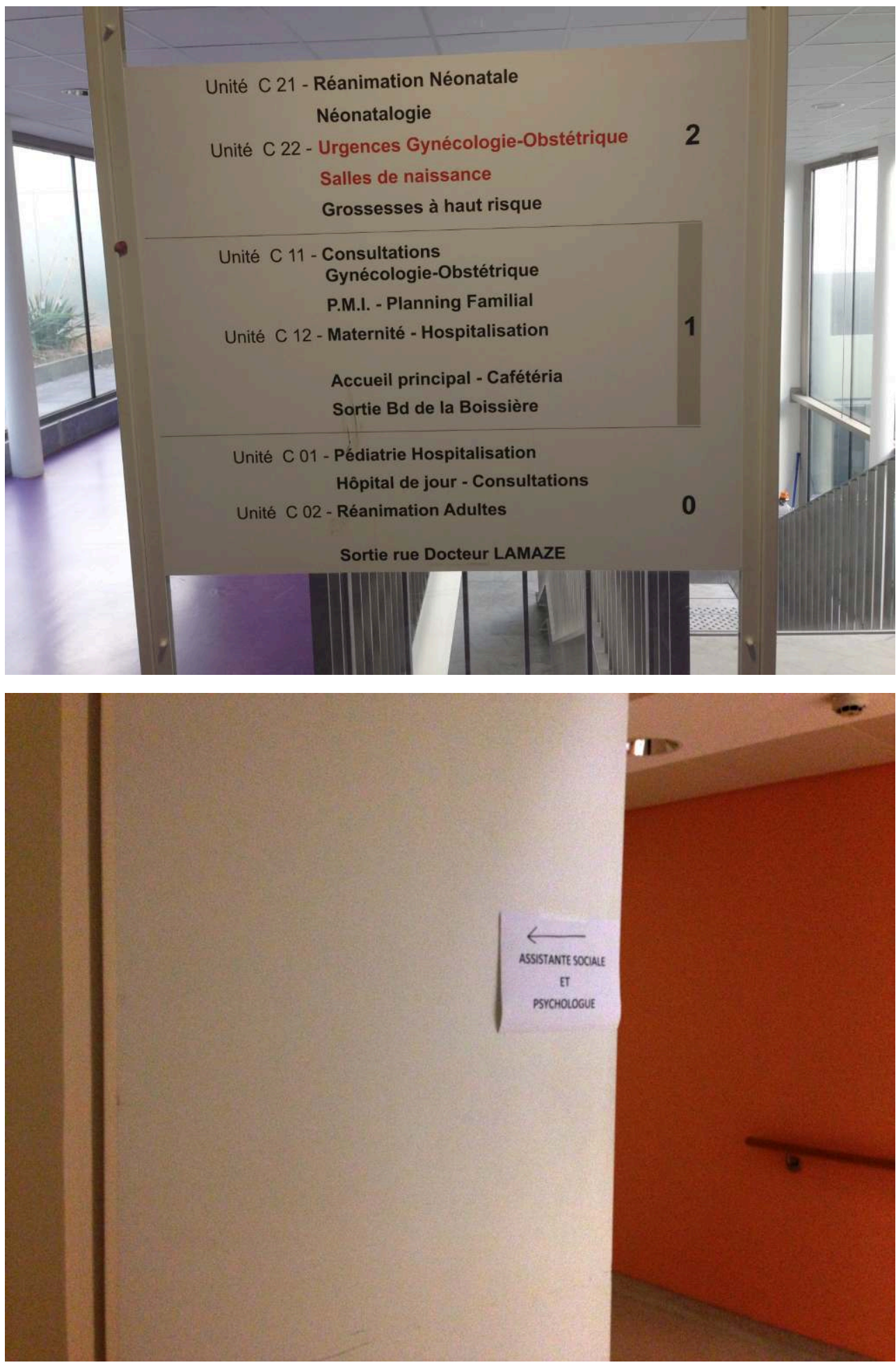
Photographies 14 et 15 : Un affichage coloré, floral permettant l'appropriation par les soignants des postes de soins à Saint-Joseph

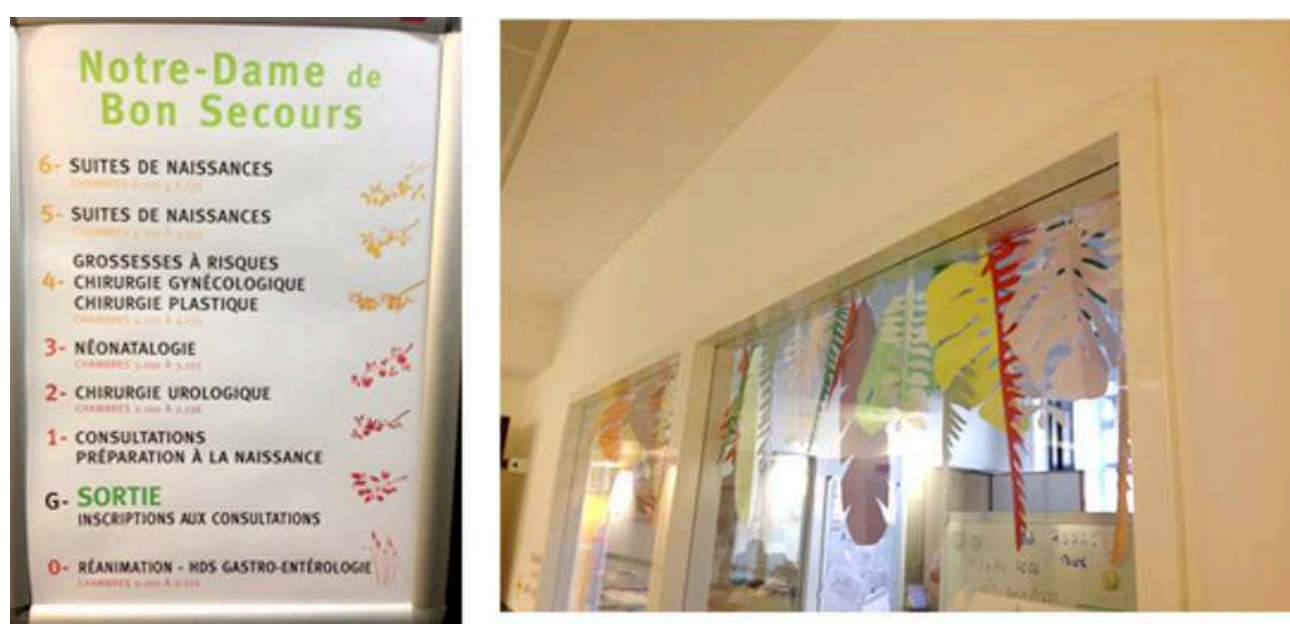

Cette fonctionnalité, ainsi que la sobriété de l'affichage s'opposent à l'affichage, plus chaleureux et travaillé, observé à Saint-Joseph. L'on peut y remarquer des éléments, reliés à la nature (photographie 14), se référant à l'idée de bien-être (calme, épanouissement, sérénité), à destination des patientes et de fait des soignants. Cette initiative, amorcée par la direction de la communication du poste de soins, a été réappropriée comme l'illustre la décoration par les professionnels de santé (photographie 15). Cela participe à la création d'un environnement approprié et la possibilité pour les soignants d'investir collectivement et personnellement cet espace.

Photographie 16 : Fauteuil d'allaitement/d'accompagnant à Saint-Joseph

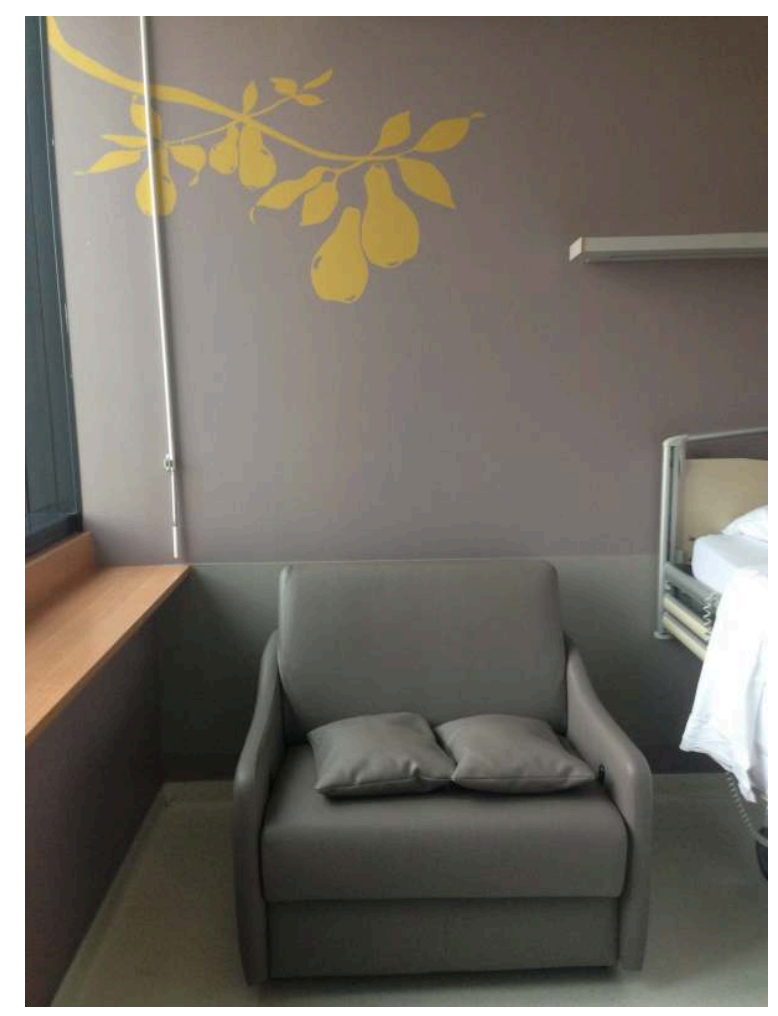


Photographie 17 : Chambre avec vue sur la tour Eiffel à Saint-Joseph

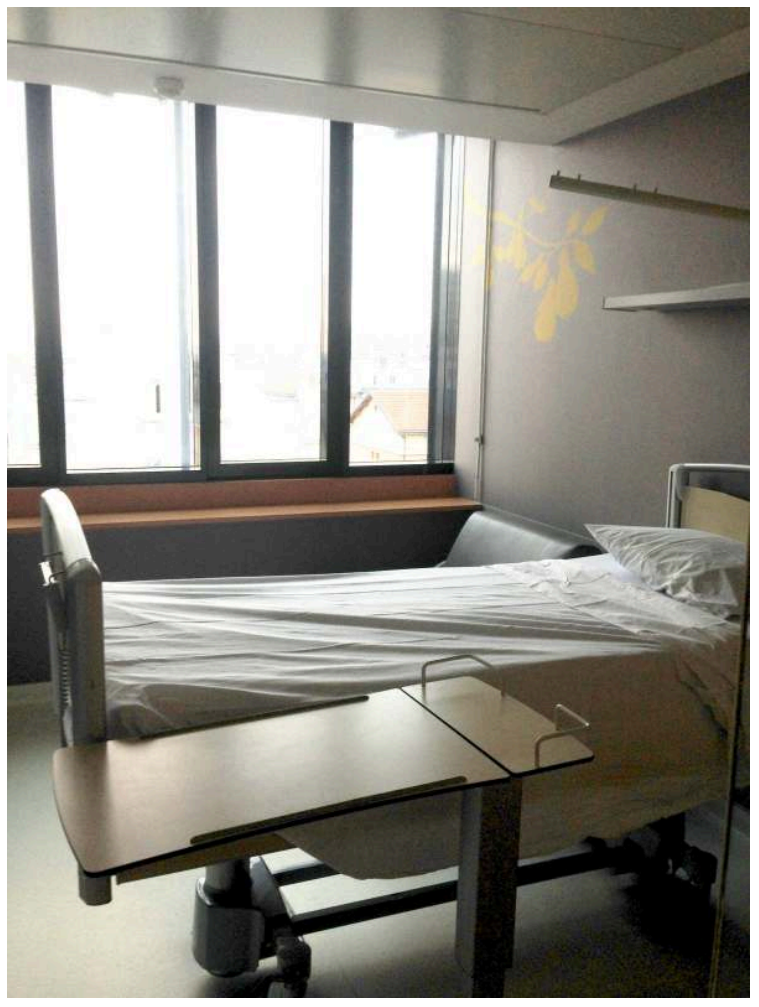

Photographie 18 : Boiseries pour l'espace nurserie à Saint-Joseph

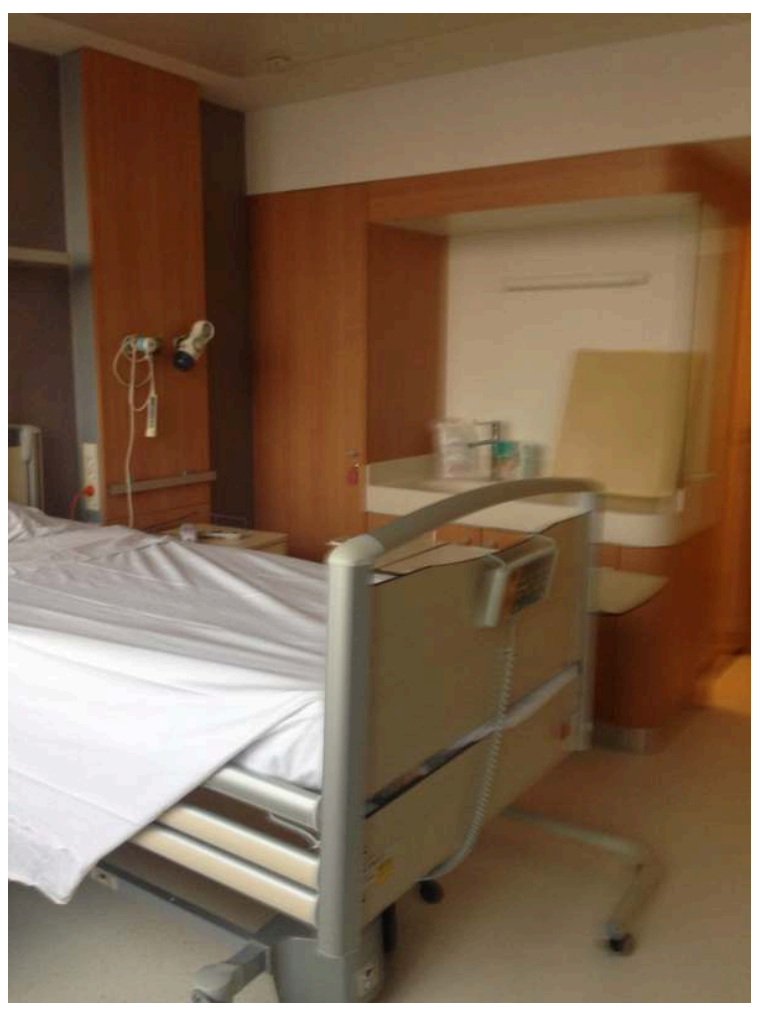


Photographie 19 : Chambre hospitalière standard à Montreuil

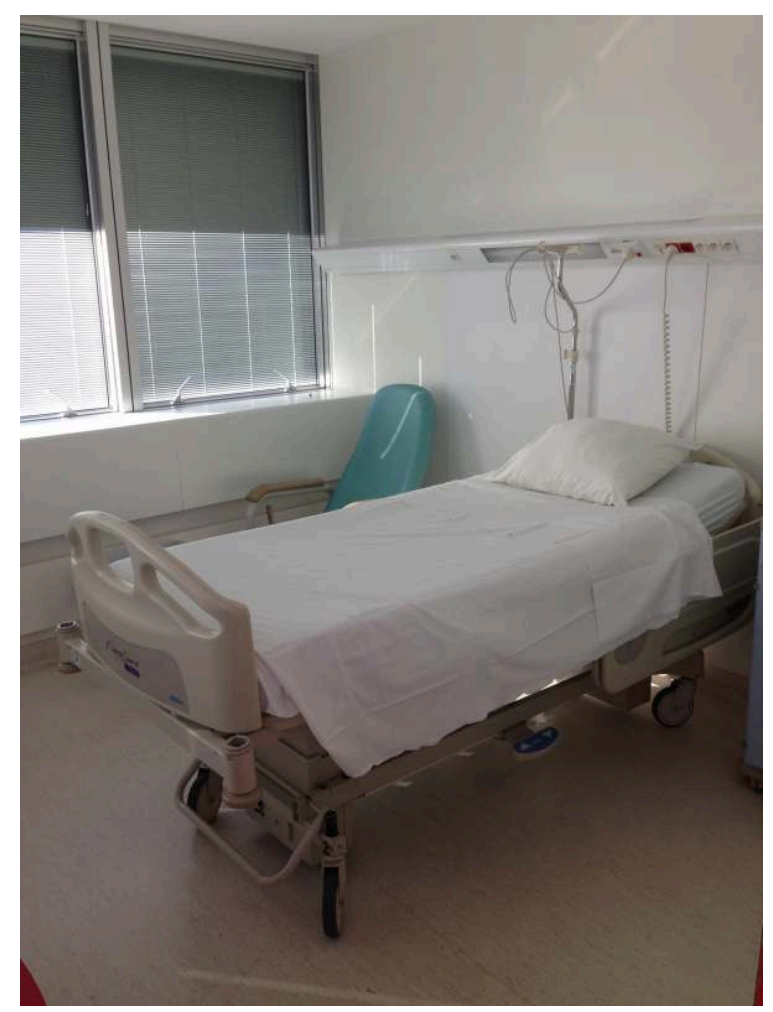

21 Les chambres de Saint-Joseph sont emblématiques d'un bâtiment et d'un mobilier pensés en vue $d u$ bien-être des patient.e.s et de leur conjoints ${ }^{13}$. Les clichés 16 à 19 montrent une chambre, à la vue agréable, au fauteuil dans lesquels les accompagnants peuvent être confortablement installés, et au cadre de décoration soigné (décorations florales et boiseries). Ce type de chambre s'oppose une fois encore à la frugalité de la chambre de la maternité de Montreuil (photographie 19), qui révèle une fonctionnalité brute, dépourvue de toute personnalisation, au fauteuil d'appoint partiellement inclinable, et à la blancheur d'une chambre toute hospitalière. 
Photographie 20 : Salles (et modalités) d'attente à Montreuil

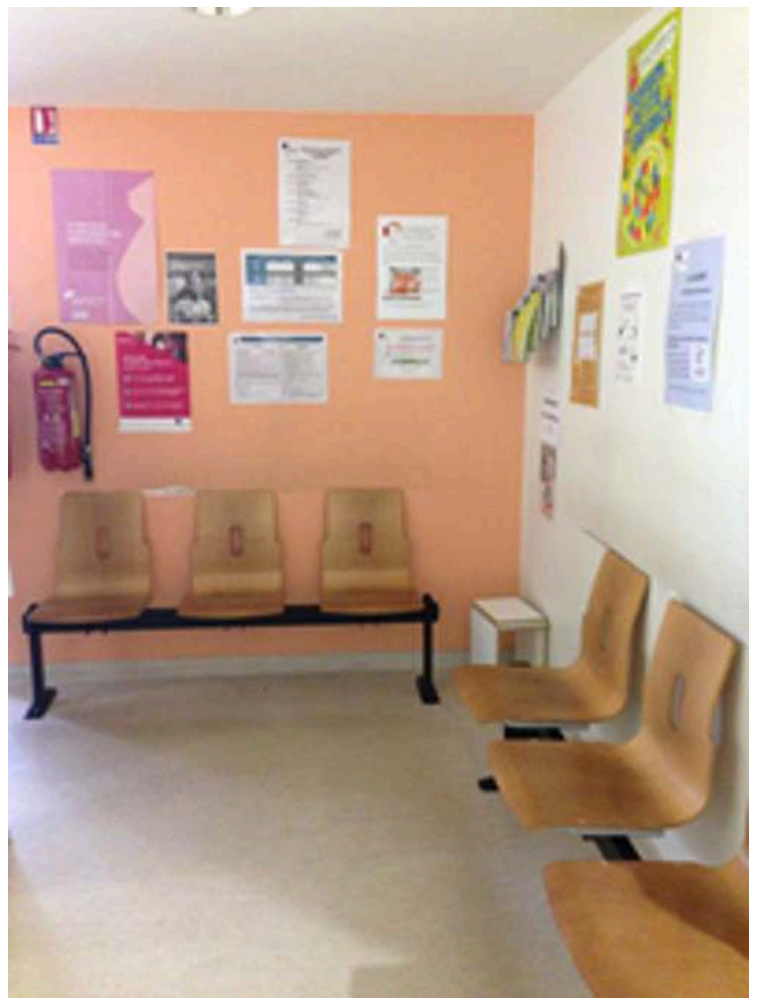

Photographie 21 : Salles (et modalités) d'attente à Saint-Joseph

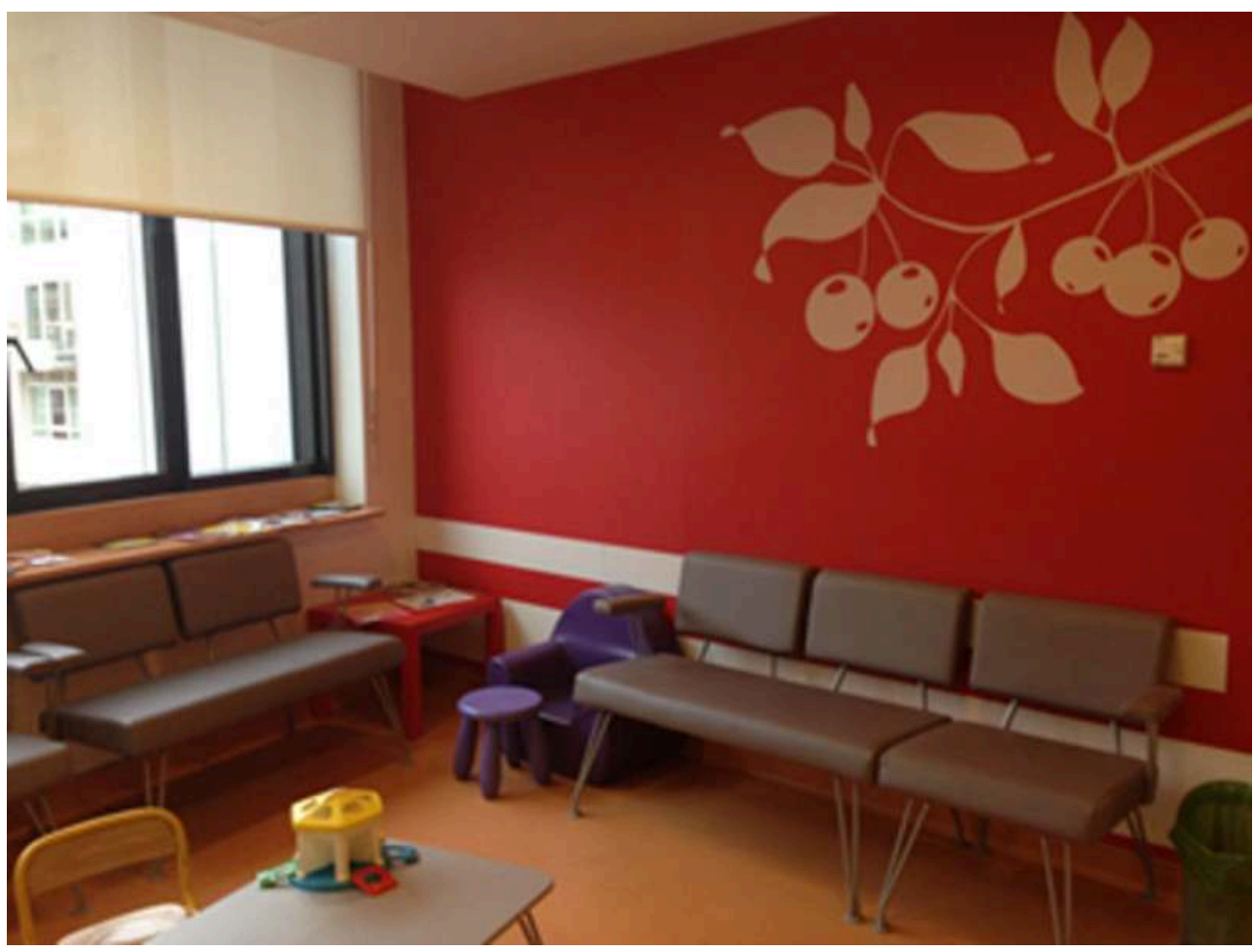

22 Dans la continuité du confort des chambres ou de l'accueil, et de la clarté de l'affichage, les espaces dédiés à l'attente renseignent également sur la facilité de l'appropriation des lieux. À Montreuil, la salle d'attente permet plus difficilement une 
attente confortable et détendue en raison de son austérité et de son aspect basique, le mobilier sobre en bois, quasi-scolaire est moins accueillant qu'à Saint-Joseph. Lors de notre visite, les femmes qui attendent sur ces chaises ne retirent pas leur manteau. De plus, l'affichage multiple, bien que signe de la mission assumée de cet hôpital public de contribuer aux campagnes de prévention, ne reste que peu visible selon la place où l'on se trouve, en même temps qu'il s'impose à la vue des patientes.

23 A l'inverse, à Saint-Joseph, l'espace d'attente est pensé pour que l'on puisse s'y projeter, s'y installer : l'espace est ouvert sur l'extérieur, les banquettes confortables invitent à poser ses affaires, un mobilier adapté aux petits est présent pour faciliter l'attente, l'accès à l'information peut être choisi par la femme si elle souhaite accéder aux prospectus (qu'elle pourra emporter) disposés le long de la fenêtre. S'oppose ici, deux tendances qui révèlent des types d'approches de prise en charge différenciés des patientes, l'une (Montreuil) propose une pluralité d'information en amont de la consultation, quand l'autre (Saint-Joseph) démédicalise l'espace d'attente.

24 La mise en comparaison de ces aspects de l'environnement physique de ces deux maternités nous renseigne sur une prise en considération en amont, par les services de communication, de direction, d'aménagement des lieux et des espaces, d'une politique différenciée à l'égard du public accueilli, mais également peut-être des types de modèles financiers et d'arbitrages de ces deux établissements ${ }^{14}$.

Dès lors il semble important pour alimenter les postulats qui pourraient être soulevés, d'appréhender dans un second temps l'environnement social au sein de ces deux maternités.

\section{L'environnement social, un enjeu de négociation de l'espace des nouveaux bâtiments accueillant soignants et patientes}

26 Concernant la dimension de l'environnement social, comme élément de l'espace thérapeutique, on entend celui-ci comme se rattachant pour " beaucoup au biopouvoiri mis en avant par M. Foucault dans ses travaux. Ceci demande une approche plus qualitative, comme par exemple des sessions de discussion engageant des soignants, des soignés ou leur entourage, pour qualifier les nuances entre ce qui relève de la sécurité pour les uns et de la surveillance pour les autres» (Vaguet, Petit, Lefebvre 2012). Plusieurs questions ont dû nécessairement être résolues lors des constructions de ces deux maternités. Par exemple, faut-il pouvoir observer les patients en permanence ou leur laisser un espace d'intimité ? Ouvrir les espaces collectifs, pour faciliter les rencontres ou élargir les espaces privés pour faciliter ce sentiment d'intimité. La somme de ces options construit un paysage social, déjà un peu appréhendé dans les éléments énoncés dans la section précédente sur l'environnement physique mais que nous pouvons discuter plus en profondeur dans cette section, notamment au regard des échanges lors des restitutions que nous avons eues avec les équipes.

$\mathrm{Au}$ sein de la maternité Saint-Joseph, les discours corroborent les observations que nous avons pu faire lors de la visite. Les équipes décrivent une ambiance agréable de travail, qui s'associe à une appropriation possible de l'environnement professionnel par les équipes, notamment en raison de la prise en considération d'éléments d'organisation du travail soignant (existence d'un système de pneumatique ${ }^{15}$ par exemple), mais aussi d'un aménagement au fil du temps du bâtiment, ouvert en 2009, pour le rendre plus fonctionnel. Celui-ci est clair (les baies vitrées sont très appréciées, 
même si les volets électriques sont extrêmement bruyants et donc très critiqués), ouvert avec de nombreux espaces d'attentes. Les discours dénotent un sentiment d'optimisation du potentiel technique d'un bâtiment à taille humaine. Les conditions de travail sont décrites comme bonnes, les unités de soins comme bien conçues. Ces aménagements et ajustements au fil de l'eau du bâtiment montrent un investissement institutionnel, notamment de la direction et des services administratifs qui permettent un dialogue avec les équipes soignantes et participent à un bon investissement des lieux par l'ensemble des professionnels.

Dans la maternité de Montreuil, dont le nouveau bâtiment émerge en 2012, l'un des premiers éléments qui nous est présenté est la création, dans les anciens locaux de la maternité, d'une crèche d'entreprise avec 40 places pour le personnel de la structure et 10 places municipales. Cet élément participe à la satisfaction de l'équipe et rappelle les enjeux initiaux de la création de cet hôpital intercommunal et sa vocation également municipale inscrite dans un bassin de population et avec un objectif de lien villehôpital. En revanche, l'espace prévu pour les chambres de gardes dans le projet initial a été affecté à un autre service, sans que des solutions alternatives satisfaisantes soient proposées dans le nouveau bâtiment. Cette situation interroge sur la connaissance et la prise en considération du travail soignant qui implique une organisation complexe des équipes autour des espaces dédiés au repos durant la garde (replis vers des espaces tiers, des chambres dédiées aux patients, ou des lits d'appoints dans les bureaux...). Elle témoigne vraisemblablement d'une absence de soutien de la direction administrative, illustrant peut-être des techniques de management moins soucieuses des conditions de travail des employés.

29 Que ce soit dans l'une ou l'autre des maternités visitées, l'organisation de l'espace nous renseigne sur la manière dont peut être conceptualisé l'hôpital comme lieu de bien-être et de soins pour les patients mais également pour les professionnels de santé.

30 La communication, notamment en vue de la promotion des deux maternités (quasi inexistante pour Montreuil et plutôt bien développée pour Saint-Joseph, sur son site internet notamment: plans, photographies, visite de la maternité en vidéo), nous renseigne également sur les stratégies différenciées pour attirer et entretenir une patientèle différente.

Photographie 22 à 24
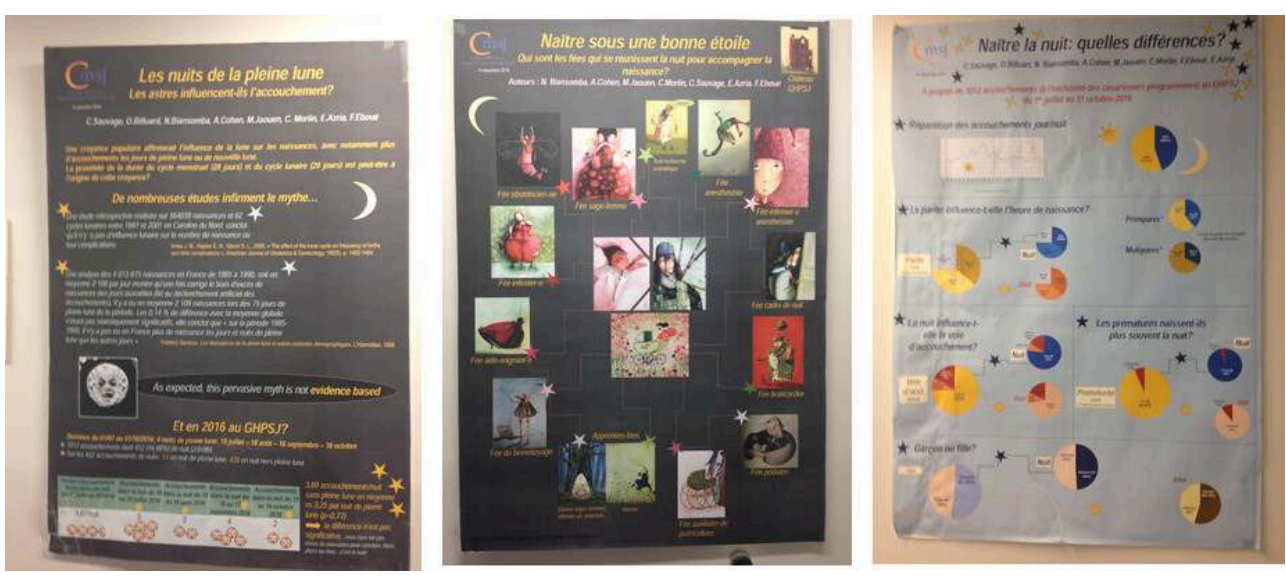

Les posters affichés dans l'espace des différentes salles de naissances montrent un lien qui se veut rassurant entre science, et intimité des patientes, en vue d'un décloisonnement du pouvoir médical et des hiérarchies au sein du service de la maternité de Saint-Joseph 
31 Comme l'illustrent les posters affichés à l'entrée de la salle de naissance de SaintJoseph, "les projections identitaires conduisent à des attentes spécifiques sur l'organisation des lieux et interrogent également sur l'image renvoyée par ces lieux» (Larceneux, 2011). Ces affichages nous renseignent sur l'exercice de décloisonnement des relations soignants et soignées mais également sur les relations hiérarchiques au sein du service. Ces posters de vulgarisation scientifique indiquent une réflexion de l'équipe de la maternité sur des sujets « connexes » qui importent aux femmes, et sont liés à leurs représentations, notamment à certaines idées reçues autour de l'accouchement : naiton plus le jour, la nuit? une nuit de pleine lune? sous quelle étoile? Cet affichage, scientifiquement décalé, présentant de manière détournée l'équipe soignante, participe à structurer un travail d'équipe et l'interaction avec la patiente.

32 Si nous ne retrouvons pas d'affichage de ce type à Montreuil, cela ne signifie pas tant une persistance des hiérarchies médicales au sein de l'équipe car l'ambiance de travail qui règne semble également conviviale bienveillante et non descendante. Cette ambiance renseigne davantage sur la nécessité ressentie de structurer le service autour d'un projet collectif, vraisemblablement implicite pour les professionnels qui travaillent dans le service public ou sur la mise en place d'actions envers un public en situation de précarité? En ce sens il relèverait du troisième versant de l'espace thérapeutique : l'environnement symbolique.

\section{Environnement symbolique}

L'environnement symbolique constitue, lui aussi, un point central de l'environnement thérapeutique. Il nécessite de passer du design à l'écoute de l'expérience du design, en allant vers les usagers des espaces (Vaguet, Petit, Lefebvre 2012). En géographie de la santé, ce domaine a surtout été renseigné dans les hôpitaux psychiatriques [Curtis 2007, 2011]. Pourtant, les attributs symboliques sont toujours décrits comme importants dans la littérature: la présence d'un mobilier personnel (personnalisé, personnalisable) pour se sentir chez soi, la possibilité de rencontrer le sexe opposé, ou au contraire la volonté de rester entre femmes ou entre hommes, sont autant d'éléments qui participent à cet environnement, comme la mise à disposition d'espaces de culte, la prise en compte de préférences ou d'interdits culinaires, la manière de s'exprimer du personnel, la possibilité de le comprendre aisément etc., l'ensemble de ces éléments concernent cette dimension symbolique. 
Photographies 25 et 26 : Entre ancrage religieux et démédicalisation de la grossesse, des symboles qui au sein des deux maternités révèlent des enjeux stratégiques. Opposition des symboliques au sein des deux maternités.

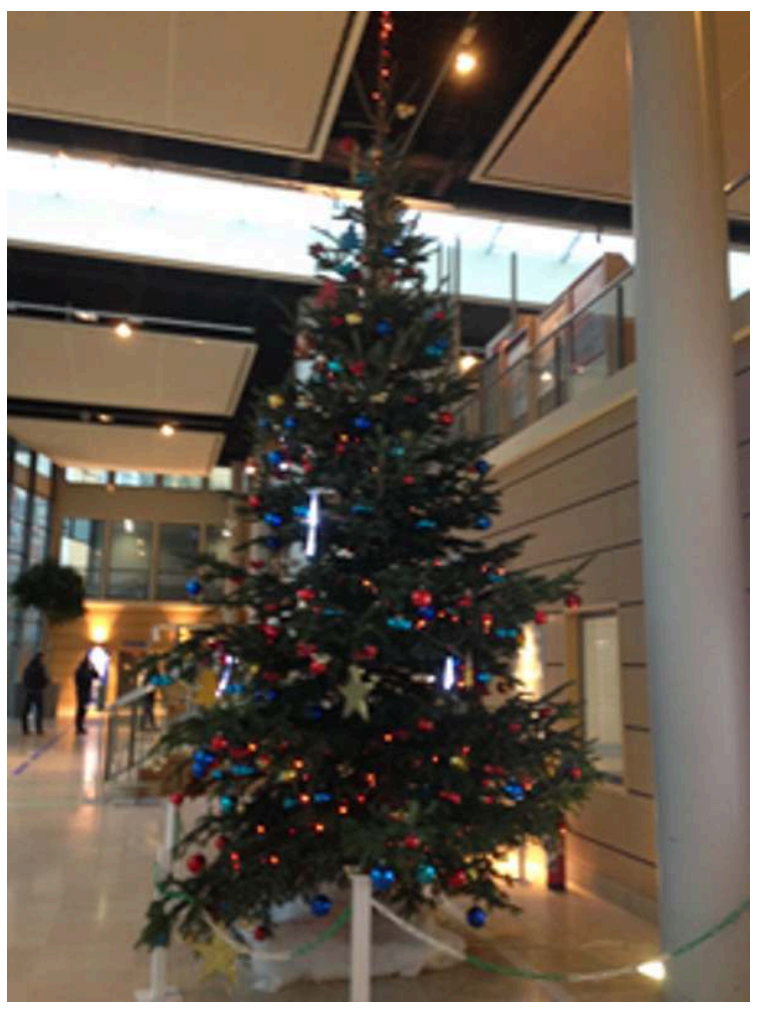




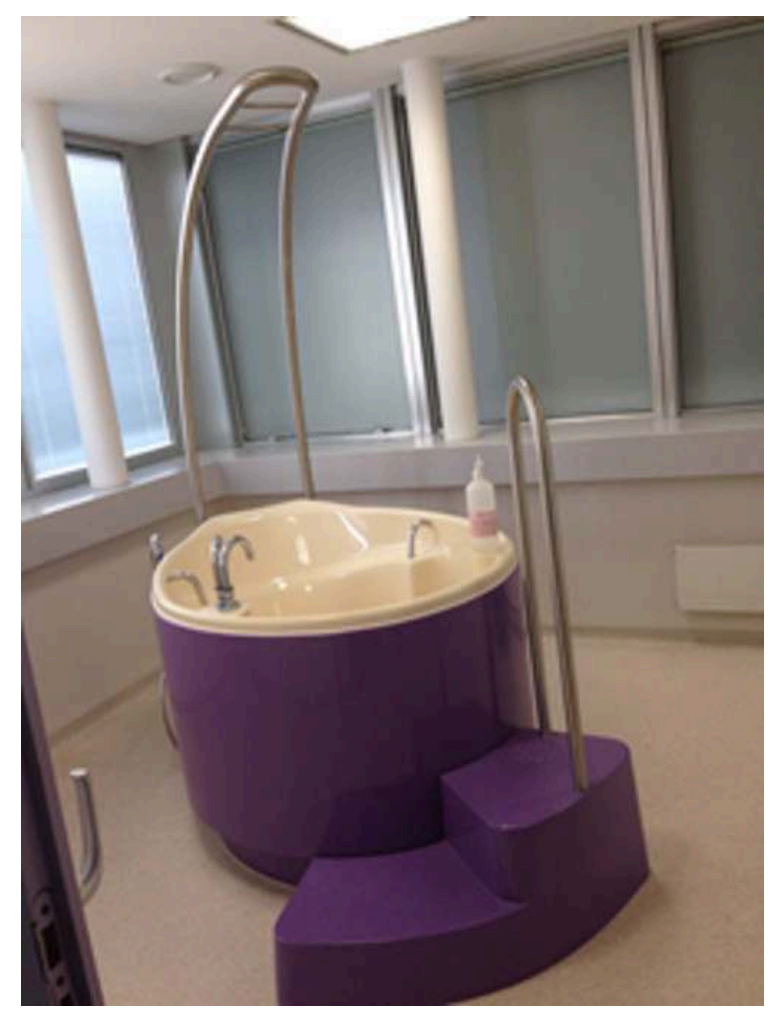

Si le sapin, n'est pas un symbole religieux, il est néanmoins fortement associé à la période de Noël, une fête chrétienne, en raison du moment de son apparition et de sa décoration dans les lieux publics. Celui de la photo doit faire plus de 6 mètres et est posté à l'entrée de l'hôpital de Saint-Joseph. Un élément du paysage intérieur de l'hôpital que l'on ne peut occulter. A Montreuil, la salle de naissance avec baignoire, pour les accouchements physiologiques dans l'eau, nous est présenté par le chef de service comme une bataille de l'équipe pour son obtention. Une bataille d'autant plus importante qu'elle vise également, dans une maternité de haute technicité (type 3) à défendre l'accouchement et son accompagnement comme pratique non systématiquement médicalisée

Le paysage symbolique apparait aussi fondamental pour les patientes que pour les soignants mais elle prend des formes très différentes selon les maternités. La symbolique au sein de cette structure est objectivée dans la pratique soignante, plus que dans l'environnement, même si quelques éléments transparaissent, comme la salle nature (baignoire dans une salle dite "nature" pour laquelle le chef de service a longuement argumenté pour en obtenir l'ouverture et promouvoir des accouchements physiologiques), ou la présence en service de néonatologie de lit kangourou (c'est-àdire de chambre d'hospitalisation mère-enfant en néonatologie). On retrouve dans le discours de l'équipe de Montreuil un vocabulaire guerrier (bataille, lutte), reprit également par le personnel de Saint-Joseph ayant par le passé travaillé dans le service public hospitalier. Comme l'évoque une sage-femme parlant de son passage à l'APHP ${ }^{16}$ : "C'est une lutte pour qu'on vous répare un volet, une lutte pendant 6 mois, (...) on lutte avec les patients, mais aussi avec des situations administratives, qui vous épuisent ».

La symbolique transcende l'espace matériel (le volet) et social (l'administration) de l'exercice professionnel, révélant une posture morale et symbolique de sa profession: mener la bataille de l'équité face à l'injustice sociale. Même si celle-ci tourne autour d'un volet, elle reflète la nécessité pour les soignants, de revendiquer un espace de soins serein et non bruyant pour les femmes, les nourrissons et eux-mêmes. Ce discours apparaît de manière récurrente dans les échanges avec le personnel de la maternité de Montreuil ; lutte quotidienne qui révèle également l'engagement des équipes du service 
public. Enoncé lors de l'exposé de restitution, cet élément a été discuté par certains professionnels de Saint-Joseph, soulignant que des difficultés sont également existantes, mais un bassin de population dissemblable entre les deux structures participe à atténuer le débat et à reconnaître la difficulté des équipes de Montreuil. Bien que Saint-Joseph soit reconnue comme conduisant une mission de service public, les discussions menées ultérieurement aux visites, interrogent systématiquement la catégorisation public/privé. Plusieurs soignants disent d'ailleurs qu'ils "se sentent public ». Ce sentiment d'appartenir, de faire fonctionner le bien public même dans un établissement privé, peut exister chez les soignants. Cette autojustification questionne la valorisation actuelle du métier de soignant, et les valeurs morales et symboliques du soin, et notamment la difficulté pour certains soignants à assumer un exercice de soins dans un établissement privé, bien qu'il soit d'utilité publique. Ce sentiment d'appartenance au public, alors que l'exercice professionnel reste de mission de service public mais dans un établissement privé, peut exister chez les soignants. Durant les discussions, d'autres soignants soulignent que leur exercicere lève néanmoins dans leur représentation d'un management privé, évoquant notamment la lettre des $\mathrm{RH}$, avec une photo quizz ancienne de l'hôpital (dont il faut trouver le pendant actuel), permettant aux personnels de la structure d'intégrer les éléments d'histoire de l'environnement symbolique des lieux (comme la visite nous l'avait fait entrevoir) ${ }^{17}$.

36 Ces éléments donnent des indications sur la qualité du travail en équipe et les postures professionnelles dans l'institution. Ces pistes sont corroborées par l'ambiance qui émane de Saint-Joseph. Pour autant, comme l'évoque une sage-femme (travaillant dans une maternité publique) et avec qui nous déambulons dans le groupe hospitalier SaintJoseph: "Ici, les patients et les soignants ont l'air épanouis (...) ce ne sont pas les mêmes patients (sous entendant, ceux auxquels elle est habituée), c'est sûr! (...) mais je ne travaillerais pas là ». Cette soignante signifie, par cette affirmation, son attachement au service public, représentant pour elle la nécessité d'exercer auprès d'un public plus mixte et en situation de précarité en invoquant le choix du lieu de son exercice (une maternité publique d'une ville populaire) comme élément de son engagement.

37 A contrario, "pour une partie de la population, notamment les classes les plus populaires, l'hôpital apparaît comme un univers hostile et non rassurant pour tout usager dépourvu de connaissances et d'argent» (Ebang Ondo, 2012). Citation interrogeant le fait que c'est justement dans les établissements publics que le plus d'efforts devraient en pratique être faits à destination de cette population, pour qu'elle puisse s'y sentir accueillie. D'autant que, dans les contextes sociaux précaires, "l'absence de repères physiques, d'indications écrites peu explicites et incompréhensibles pour les non-alphabétisés, explique que les nouveaux arrivants soient souvent perdus » (Nikiema, 2014). 
Photographies 27 et 28 : L'hôpital de Saint-Joseph et ses symboles historiques, avec ses coursives extérieures et également un symbole identitaire, ici catholique, avec la vue sur la chapelle représentant l'articulation entre modernité et mémoire des lieux

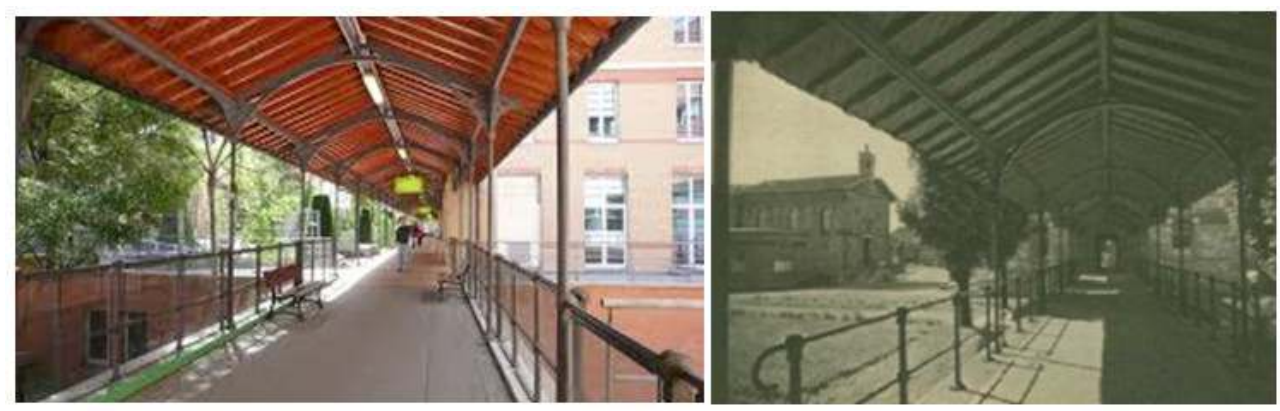

Source : @ site internet du Groupe hospitalier Paris Saint-Joseph

38 Ce sentiment d'appartenance à un établissement participe à l'idée d'exercer dans un établissement ancien, chargé d'histoire. Cela se lit dans le paysage hospitalier, ponctué de signes, de constructions à la charge symbolique forte qui participent à l'impact thérapeutique des aménagements sur les patients et les soignants, dans un jeu de frontières/barrières et d'ouverture. Mais il faut bien noter que chaque signe symbolique reste contingent. Ainsi la présence de vigiles à l'entrée d'un hôpital peut apparaître sécurisante pour le patient, ou inversement anxiogène. Il en va de même pour la continuité historique dans le bâti qui fait perdurer une unité et une identité symbolique (notamment religieuse) forte à l'établissement, liant également d'une part un respect dans l'architecture moderne de l'hôpital de l'histoire du site, et d'autre part l'entité chrétienne et l'hospice, deux symboles identitaires forts, qui s'opposent à la sobriété technique du service public.

39 La (non) signalétique (un code couleur au sol, ou inversement les coursives extérieures de Saint-Joseph, ou encore l'absence de signalétique à Montreuil) pour accéder à un service, à une chambre... peuvent favoriser et simplifier la déambulation dans l'espace hospitalier.

40 Ces différents éléments participent à développer ou entraver la création d'un lien affectif avec le lieu, qui dans le cas présent est particulièrement sensible puisque c'est celui de la mise au monde d'un enfant. Dans son historicité, la chapelle, construite au centre de l'hôpital pavillonnaire, favorise la reconnaissance du lieu et une intimité pour la communauté catholique, mais peut également participer à un aspect communautaire, n'autorisant pas/peu cette appropriation de l'espace du soin par une population appartenant à une autre communauté religieuse.

41 Tous ces éléments participent à une «bonne qualité de travail » comme l'évoque une soignante, sage-femme cadre de Saint-Joseph lors de la visite et en témoignent les discussions, autour du management des équipes, qui décrivent peu de conflictualité entre les personnels administratifs et les soignants de la maternité de Saint-Joseph, en regard de ce qu'ils ont pu connaître dans le public pour ceux qui y ont travaillé. Il semble ici exister un dialogue entre la direction et l'univers des soignants. On constate également une conscience des soignants de voir l'établissement à l'équilibre financier, avec notamment la mémoire de la fusion des différents hôpitaux et des plans sociaux qui y ont été associés, éprouvants pour les équipes, contraintes que ne connaissent pas sous cette forme les équipes du service public, soumises à des difficultés toutes autres, notamment d'accueil inconditionnel, et avec, si ce ne sont des moyens moindres, des 
tutelles différentes et des arbitrages et justifications budgétaires différenciées. À l'inverse, à l'issue de la présentation de la synthèse de nos observations lors des visites, les discussions issues des restitutions par l'équipe à la maternité de Montreuil ont surtout portées sur les différences de moyens entre les deux maternités - la leur étant présentée dans le discours des soignants de Montreuil comme moins bien dotée, du fait $\mathrm{du}$ financement (uniquement public) et du public accueilli (le bassin de population étant d'origine beaucoup plus populaire et le public plus vulnérable qu'à Saint-Joseph).

Photographies 29 à 32 :
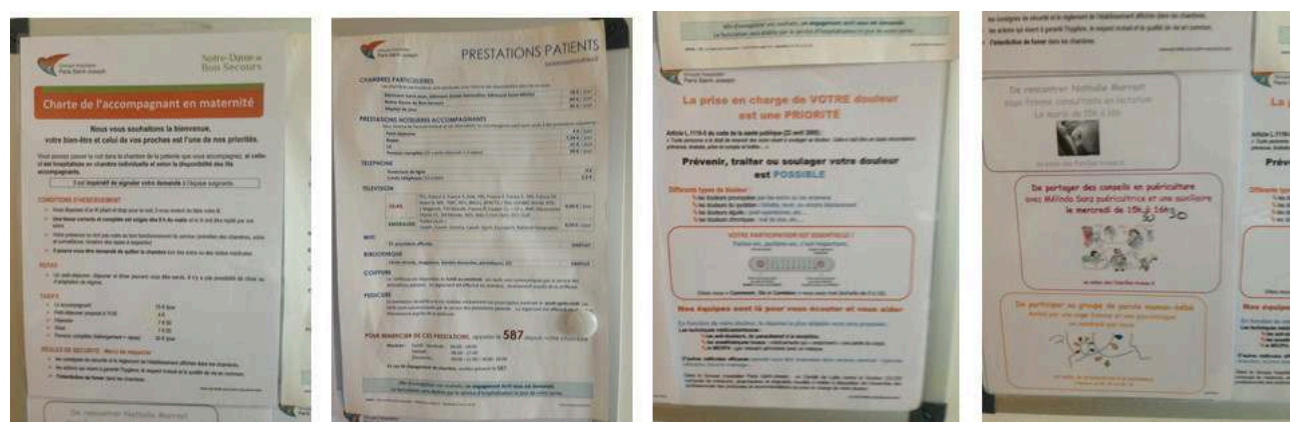

Affichage à Saint Joseph d'une prise en charge individuelle : vers une « médecine personnalisée » (Charte de l'accompagnement en maternité et prestation pour les patients (payantes), Gestion de la douleur atelier bébé)

Photographies 33 à 35 :
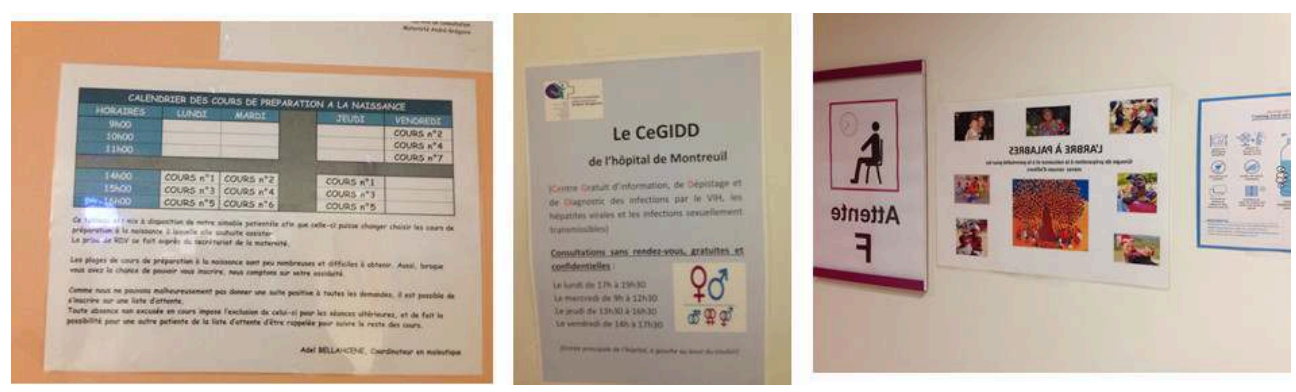

A Montreuil, l'accueil est à visée collective et opérationnelle. L'affichage montre une prise en considération des questions de médecine préventive et une orientation vers des parcours pour les femmes correspondant aux recommandations de la Haute Autorité de Santé

La différence de patientèle participe à une forme de prise en charge opposant les deux maternités. Nos observations nous renseignent sur un affichage pour des ateliers collectifs (photographie 33), en lien avec les recommandations des instances sanitaires, qui rappellent le contexte social environnant et de la population accueillie mais qui renvoie aussi cette population à certaines de ses vulnérabilités (CEGIDD) (photographie 34) ou à des assignations identitaires (besoin de soutien, de groupe de parole : arbre à palabre (photographie 35)). Elles s'opposent à la prise en charge affichée à Saint-Joseph plus personnalisée et individuelle (photographies 29 à 32).

\section{Conclusion}

43 L'opposition théorique public/semi-privé n'est pas toujours opérationnelle. En fait, bien des aménagements du privé ne sont que de petits ajouts qui pourraient très 
facilement être faits dans le public mais ne le sont pas toujours pour des raisons tant budgétaires que de management. Malgré l'écart important dans les publics accueillis (en termes de capital économique et culturel), les deux équipes soignantes retirent de la satisfaction et de la fierté de leur travail, avec, peut-être, à la maternité de Montreuil, en plus, la conscience d'être encore plus utile socialement. Si on ne peut pas clairement objectiver des prises en charge différenciées sur la base d'échanges avec des équipes soignantes et l'observation de leurs lieux de travail, on pressent néanmoins une tendance à cette différenciation plurifactorielle.

A l'issue de ces deux visites, en dépit des limitations de cette étude, on constate que l'entrée théorique par les trois versants de l'espace thérapeutique est pertinente pour documenter les hôpitaux en tant qu'espace de bien-être. Si l'exercice soignant et l'engagement qui l'accompagne se retrouvent sur les deux sites, le bassin de population, le management et la mission de ces établissements diffèrent. Le soin ne peut être identique voire équitable selon les locaux, leur agencement et les symboles qu'ils portent. Ces constats renvoient plus largement aux très fortes disparités territoriales d'offre de soins existantes en France, qui à l'extrême font de certaines zones des déserts médicaux, même en Île-de-France. L'appropriation des lieux à SaintJoseph s'oppose aux lieux opérationnels destinés à une médecine collective à Montreuil. L'urgence de la mission de service publique, et l'engagement important des soignants face au risque d'inégalité d'accès à tous de manière indifférenciée, font passer au second plan la personnalisation auprès des plus vulnérables. Dès lors, comme le souligne une sage-femme de l'Unité d'accompagnement personnalisé qui regrette que "toutes les patientes ne puissent finalement pas être prises en charge de cette manière ", (sous-entendu comme au sein de l'Unité d'Accompagnement Personnalisée mise en place à Montreuil en 2018). On retrouve un engagement à Saint-Joseph également mais avec une offre de service supplémentaire, admise par les soignants du privé. En effet, les enjeux financiers semblent pris en considération par les soignants du privé, ils sont plus sévèrement critiqués par les soignants du public. Certains d'entre eux ne souhaitent pas proposer l'offre de chambre simple pour mettre toutes leurs patientes "à la même enseigne ", particulièrement dans la mesure où la nouvelle maternité de Montreuil ne dispose que de chambres individuelles ${ }^{18}$.

\section{BIBLIOGRAPHIE}

Briand P., Pary A., 2011. L'hôpital : un indicateur de solidarité sociale. Centrale sanitaire suisse romande, cahier $\mathrm{n}^{\circ} 5$, p. 3.

Coldefy M., 2010, De l'asile à la ville : une géographie de la prise en charge de la santé mentale en France. Thèse de géographie, Université Paris 1 Panthéon Sorbonne. https://tel.archivesouvertes.fr/tel-00498772

Clémençon AS, 2002. Le rôle de l'institution hospitalière dans l'urbanisation : le cas des Hospices civils de Lyon. Actes du colloque Hôpital, urbanisme et architecture, Grand Lyon Mission Prospective et Stratégie d'agglomération, p13-14 
Curtis S., Gesler W., Fabian K., Francis S., Priebe S., 2007. Therapeutic landscapes in hospital design: a qualitative assessment by staff and service users of the design of a new mental health inpatient unit, Environment and Planning C: Government and Policy ,volume25, p591-610. DOI: $10.1068 / \mathrm{c} 1312 \mathrm{r}$

Curtis S., 2016, Space, Place and Mental Health, Routledge, 1 avr. 2016, 316 pages

Curtis S., Gesler W., Priebe S. \& Francis S., 2009; New spaces of inpatient care for people with mental illness: A complex 'rebirth' of the clinic?, Health \& Place, Volume 15, Issue 1, March 2009, Pages 340-34

Ebang Ondo E., 2012. Perception de l'hôpital public et offre de soins de santé au Gabon : analyse des enjeux des interactions entre personnels et usagers du Centre Hospitalier de Libreville (CHL). Bulletin Amades , n 85, 6 p. https://doi.org/10.4000/amades.1398

Fermand C., 2002. Pour une histoire urbaine et architecturale de l'hôpital. Cahier Millénaire 3, Actes du colloque " Hôpital, urbanisme et architecture », 3 avril 2002, Hôtel de la Communauté urbaine de Lyon, p.10-11.

Fleuret S., 2012. L'évolution des savoirs en santé et la place de la médecine dans la société, dans les territoires et dans la ville. Espaces et sociétés , UMR 6590 CNRS, Universitéd'Angers, 33, p.43-55.

Gesler W., 1992. Therapeutic landscapes: medical issues in light of the new cultural geography. Social science and medicine, vol. 34, $\mathrm{n}^{\circ} 7, \mathrm{pp} 735-746$.

Gesler W. M., 1998: Bath's reputation as a healing place. In Kearns R. and Gesler W., editors, Putting health into place,Syracuse, NY:Syracuse University Press,17-35.

Gesler W., Belle M., Curtis S., Hubbard P., Francis S., 2004. Therapy by design: evaluating the UK hospital building program. Health and place , 10, p. 117-128.

Gesler et Kearns 2002, Culture/Place/Health, Psychology Press.

Gesler et al., 2004, Therapy by design: evaluating the UK hospital building program,Health Place. 2004 Jun; 10(2):117-28.

Hoyez, A.-C., 2005; L'espace-Monde du Yoga. Une géographie sociale et culturelle de la mondialisation des paysages thérapeutiques. Géographie. Université de Rouen, 2005. Français.l tel-00011237v1 >

Hoyez A.-C., Gasquet-Blanchard C., 2019, Lieu(x), 26 mots pout situer la santé, Abécédaire de la santé et des territoires, Materiologiques

Labasse, J., 1980, L'Hôpital et la ville, Géographie hospitalière, Paris, Hermann, 241 p.

Larceneux F., 2011. J'habite donc je suis. Etudes foncières , n²3-26, 6p.-Nikiema A., « Approche spatiale d'un centre hospitalier, le CHU pédiatrique de Ouagadougou (Burkina Faso) », EchoGéo [Online], 30 | 2014, Online since 11 December 2014, connection on 02 October 2016. URL : http:// echogeo.revues.org/14046 DOI:10.4000/echogeo.14046

Vaguet A., Lefebvre B., Petit M.,2012, Lieux de soins et paysages thérapeutiques. Du concept à la méthode. (Health care places and therapy landscapes. From concept to method). In : Bulletin de l'Association de géographes français, 89e année, 2012-2. La géographie de la santé en France. pp. 214-223; DOI:10.3406/bagf.2012.8258

Granier P., 2002, Une approche multiscalaire des lieux hospitaliers : l'exemple du CHRU de Tours, Norois, T 49, n¹89, p.5-16 https://www.persee.fr/doc/noroi_0029-182x_2002_num_189_1_7050 Le Mandat, M, 1989, Prévoir l'espace hospitalier, Berger-Levrault, 665 p. 
Tantchou J., 2017, En Afrique, la matérialité du soin au coeur des tensions soignants-soignés ? in Sciences Sociales et Santé $n^{\circ} 4$ (volume) DOI:10.3917/sss.354.0069

\section{NOTES}

1. Labasse J., 1980, L’Hôpital et la ville, géographie hospitalière, Hermann, Paris, 240 p

2. Augé M., 1992, Non-lieux Introduction à une anthropologie de la sur-modernité, Seuil, 160p

3. Les références aux auteurs sont ajoutées dans le glossaire (paysage thérapeutique en note de fin)

4. La distinction entre public et privé peut-êtreprésentée dans le texte en raison des statuts différenciés de ces maternités (ESPIC-Etablissement de santé, privé d'intérêt collectif-pour la maternité de Saint-Joseph et maternité d'un Centre hospitalier intercommunal public à Montreuil) et la manière dont cet élément de distinction peut faire sens pour les quipes, particulièrement celles de la maternité publique ou ceux ayant travaillé préalablement au sein du service public.

5. «Composé de plusieurs bâtiments pouvant évoluer dans le temps et pouvant être dispersés à travers la ville » in Bridgman, R.F., 1963, L'hôpital et la cité, Les Editions du Cosmos, 347 p.

6. Bien que l'on note un phénomène de gentrification très avancée dans cette ville, ce front n'atteint pas (encore) le quartier enclavé où se situe l'hôpital.

7. Il s'agit d'un ESPIC - un Etablissement de santé privée d'intérêt collectif

8. Cette formule reste anecdotique dans l'activité de la maternité et ne concerne que quelques patientes par an. Même si ce n'est pas le cas à la maternité de Montreuil, ce type d'offre se développe de plus en plus dans certaines maternités publiques, montrant la progression de la privatisation de certains pans du service public.

9. Les attentes et inquiétudes autour de cet aménagement sont d'ailleurs importantes pour les équipes de la maternité de Montreuil : cette accessibilité induira-t-elle une fuite des patient.e.s de classe moyenne vers Paris ou parallèlement une recrudescence de patient.e.s toujours plus en situation de précarité ?

10. Quartier situé au Sud de la Mairie de Montreuil et parmi les plus gentrifiés de la ville car accessible en métro et jouxtant la capitale

11. L'information ne nous a pas été transmise concernant la maternité de Saint-Joseph

12. L'ensemble des photographies sont des clichés de terrain de C. Gasquet-Blanchard, sauf si un crédit autre est précisé.

13. Présence d'une chauffeuse dépliante pour un repos, utile après la nuit de l'accouchement...

14. L'existence d'une fondation du groupe hospitalier Saint-Joseph permet la collecte de dons en parallèle des recettes générées par l'activité hospitalière à Saint-Joseph (recette de la Caisse Primaire d'Assurance Maladie) et des subventions de l'Agence Régionale de Santé. À Montreuil, l'hôpital est marqué par le versement d'aides de l'Agence régionale de Santé île-de-France, notamment durant une période de difficulté du Centre hospitalier intercommunal de Montreuil (entre 2012 et 2014) à la suite d'un 
placement sous administration provisoire en raison d'un déclin important d'activité. Cette situation est aujourd'hui résolue.

15. Le système de pneumatique permet grâce à un réseau de tuyau dans l'ensemble du bâtiment d'envoyer et de recevoir dans des cartouches sécurisés et répondant aux normes d'hygiènes, des messages, prélèvements, etc.

16. L'APHP (ou Assistance publique - Hôpitaux de Paris) est l'établissement public de santé français qui exerce le rôle de centre hospitalier régional pour Paris et une partie de l'Île-de-France, notamment la petite couronne, il regroupe plus de 39 établissements et accueille plus de 8 millions de patients par an.

17. Si ce type de communication interne existe aussi dans des Groupes hospitaliers de l'APHP et des Groupes hospitaliers de province, il n'a pas été évoqué par les équipes de la maternité de Montreuil

18. Cela relève pour les soignants, qui évoquent le séjour en maternité aux parturientes, d'un non-sens dans la prise en charge proposée. L'administration hospitalière demande aux soignants de proposer une chambre simple (surfacturée), alors même que ces chambres individuelles constituent la majorité de «l'offre» et que les femmes ont donc toutes les chances de s'y retrouver de fait. Pour les soignants de Montreuil, pas de surfacturation en chambre simple, puisqu'elles le sont toutes déjà.

\section{NOTES DE FIN}

i. Paysage thérapeutique : au début des années 1990, en évoquant des lieux qui facilitent la guérison des patients (Epidaure, Lourdes, Bath) W. Gesler développe le concept de paysage thérapeutique, qui convoque une dimension empirique et de géographie sociale et apporte beaucoup à la géographie de la santé. Le concept a été ensuite réapproprié par un grand nombre d'auteurs (Kearns \& Gesler 1998, Gesler, Bell, Curtis, Hubbard \& Francis, 2004, Curtis, Gesler, Priebe \& Francis, 2009) avec l'idée d'interroger le rôle des lieux de soins dans la guérison, notamment dans le domaine de la psychiatrie (Curtis, 2010) ou encore en service de soins palliatifs, de médecines alternatives (Hoyez, 2015). Il peut être synthétiser en trois composantes des paysages thérapeutiques contribuant à caractériser ce qui constitue le bien-être dans un environnement thérapeutique : les éléments physiques, sociaux et symboliques et que le concept de paysage permet d'aborder de manière commune.

ii. Le biopouvoir est utilisé ici, comme concept définit et entendu au sens ou l'emploi Michel Foucault, et issue du concept de biopolitique participant à définir comment les individus, leur état de santé et de manière plus générale leur santé, peuvent être au centre d'enjeux de luttes politiques et de stratégies économiques et alors utilisés à des fins capitalistiques et néolibérales. 


\section{RÉSUMÉS}

En mobilisant le concept de paysages thérapeutiques, nous proposons de nous interroger sur l'agencement des lieux de soins et leur appropriation par les professionnels de santé dans le contexte spécifique de la prise en charge de la grossesse en maternité. La méthodologie de cette recherche repose sur la visite commentée et collective de deux maternités franciliennes de statut différent: la maternité du Groupe Hospitalier Saint-Joseph, établissement de santé privé d'intérêt collectif et celle du Centre hospitalier intercommunal public de Montreuil. Les résultats de cette recherche montrent que l'entrée théorique par les trois versants de l'espace thérapeutique s'avère pertinente pour documenter les hôpitaux en tant qu'espace de bien-être. Si l'exercice soignant, et l'engagement qui l'accompagne, se retrouvent sur les deux sites, le bassin de population, le management et la mission de ces établissements diffèrent. Le soin ne peut être identique voire équitable selon les locaux, leur agencement et les symboles qu'ils portent.

Thanks to the "cultural turn", medical geography has produced a great deal of work on health, taking the health care places as an entry point (Gesler \& Kearns, 2002, Curtis, 2004). The purpose was to give them a broad scope that questioned the place of identity, human experience, body, environment and culture. Thus, places that had a reputation for caring, affecting health, and healing, have been documented by researchers and conceptualized under the term of therapeutic landscapes (Gesler, 2002). English-speaking researchers have thus published extensively in this direction. They ask how the design of a care space can interact with the relational dimension of the caregivers. But also how the use of places could claim to have a therapeutic dimension, or even improve the quality of care and the quality of life of the sick people living in these places (Gesler et al., 2004).

More: here

INDEX

Index géographique : Île-de-France

Mots-clés : paysages thérapeutiques, maternité, soins, bien-être

\section{AUTEURS}

\section{CLÉLIA GASQUET-BLANCHARD}

Département des Sciences humaines, sociales et du comportement de l'EHESP ; UMR CNRS 6590, Espaces et Sociétés, Laboratoire Rennes 2

\section{ALAIN VAGUET}

Université de Rouen, Laboratoire IDEES, CNRS. Respectivement Président et secrétaire de la commission Géographie de la santé du Comité National Français de Géographie

\section{VÉRONIQUE LUCAS-GABRIELLI}

Respectivement Président et secrétaire de la commission Géographie de la santé du Comité National Français de Géographie, Institut de Recherche et de Documentation en Economie de la santé (IRDES) 


\section{BRUNO RENEVIER}

Service de gynécologie obstétrique, CHI André Grégoire, Montreuil, France

\section{ELIE AZRIA}

Maternité, Groupe Hospitalier Paris Saint-Joseph, Paris, France. Université de Paris, CRESS (Obstetrical, Perinatal and Pediatric Epidemiology Research Team, EPOPé), INSERM, INRA, F-75004 Paris, France 